\title{
Lymphatic endothelial cells are a replicative niche for Mycobacterium tuberculosis
}

\author{
Thomas R. Lerner, ${ }^{1}$ Cristiane de Souza Carvalho-Wodarz, ${ }^{2}$ Urska Repnik, ${ }^{3}$ Matthew R.G. Russell, ${ }^{4}$ Sophie Borel, \\ Collin R. Diedrich, ${ }^{5}$ Manfred Rohde, ${ }^{6}$ Helen Wainwright, ${ }^{7}$ Lucy M. Collinson, ${ }^{4}$ Robert J. Wilkinson, $, 1,5,8$ \\ Gareth Griffiths, ${ }^{3}$ and Maximiliano G. Gutierrez ${ }^{1}$
}

\begin{abstract}
'Host-pathogen interactions in tuberculosis laboratory, The Francis Crick Institute, Mill Hill Laboratory, London, United Kingdom. ${ }^{2}$ Department of Drug Delivery, Helmholtz Institute for Pharmaceutical Research Saarland, Helmholtz Centre for Infection Research, Saarland University, Saarbrücken, Germany. ${ }^{3}$ Department of Biosciences, University of Oslo, Blindernveien, Oslo, Norway. . ${ }^{4}$ lectron Microscopy Science Technology Platform, The Francis Crick Institute, Lincoln's Inn Fields Laboratory, London, United Kingdom. ${ }^{5}$ Clinical Infectious Diseases Research Initiative, Institute of Infectious Disease and Molecular Medicine, University of Cape Town, Observatory, South Africa. ${ }^{6}$ Central Facility for Microscopy, Helmholtz Centre for Infection Research, Braunschweig, Cermany. . Department of Anatomical Pathology, University of Cape Town Faculty of Health Sciences and National Health Laboratory Service, Groote Schuur Hospital, Cape Town, South Africa. ${ }^{8}$ Department of Medicine, Imperial College London, London, United Kingdom.
\end{abstract}

\begin{abstract}
In extrapulmonary tuberculosis, the most common site of infection is within the lymphatic system, and there is growing recognition that lymphatic endothelial cells (LECs) are involved in immune function. Here, we identified LECs, which line the lymphatic vessels, as a niche for Mycobacterium tuberculosis in the lymph nodes of patients with tuberculosis. In cultured primary human LECs (hLECs), we determined that M. tuberculosis replicates both in the cytosol and within autophagosomes, but the bacteria failed to replicate when the virulence locus RD1 was deleted. Activation by IFN- $\gamma$ induced a cell-autonomous response in hLECs via autophagy and NO production that restricted $M$. tuberculosis growth. Thus, depending on the activation status of LECs, autophagy can both promote and restrict replication. Together, these findings reveal a previously unrecognized role for hLECs and autophagy in tuberculosis pathogenesis and suggest that hLECs are a potential niche for M. tuberculosis that allows establishment of persistent infection in lymph nodes.
\end{abstract}

\section{Introduction}

Mycobacterium tuberculosis, a pathogen that primarily infects the lungs, can disseminate via the lymphatic system to virtually any organ of the human body. Rates of extrapulmonary tuberculosis have increased dramatically in the last decade, reaching up to $20 \%$ of the cases. This disease has become an important clinical problem, in particular in $\mathrm{HIV}^{+}$patients (1). The most common extrapulmonary sites are the lymph nodes, and viable bacteria can be recovered from these organs (2). However, it is unclear where M. tuberculosis resides in the lymph nodes.

The host-pathogen interactions in tuberculosis are complex, and several host and pathogen factors contribute to the pathogenesis of this disease (3). Experimental infections and genetic studies of susceptibility to mycobacteria have stressed that IFN- $\gamma$ is a key cytokine for control of M. tuberculosis, in particular by IFN- $\gamma$-activated macrophages (4-6). However, several cells of the immune system respond to IFN- $\gamma$, and nonmyelocytic responders to IFN- $\gamma$ are required for the control of the infection in the mouse model of tuberculosis (7). M. tuberculosis lacks some classical virulence

Authorship note: T.R. Lerner and C. de Souza Carvalho-Wodarz are co-first authors. Conflict of interest: The authors have declared that no conflict of interest exists. License: This work is licensed under the Creative Commons Attribution 4.0 International License. To view a copy of this license, visit http://creativecommons.org/ licenses/by/4.0/

Submitted: June 19, 2015; Accepted: January 4, 2016

Reference information: / Clin Invest. 2016;126(3):1093-1108. doi:10.1172/JCI83379. factors present in most human bacterial pathogens, such as toxin production (8). Despite this, it is clear that a genetic locus known as the region of difference 1 (RD1) region encoding a type VII secretion system (ESX-1) is required for growth of M. tuberculosis in macrophages and epithelial cells and for replication in mice (9-12). Bacterial components encoded in the RD1 region are involved in the cytosolic localization of M. tuberculosis in myelocytic cells, in activation of the DNA-sensing pathway in the cytosol, and in induction of host cell death after infection (13-15).

Although M. tuberculosis primarily infects myelocytic cells, the pathogen can infect numerous cell types in vitro, and bacterial components can be found in other cell types of many organs in humans (16). In this regard, lymphatic endothelial cells (LECs), which are functionally different from vascular endothelial cells $(17,18)$, are emerging as critical components of the innate and adaptive immune response to infection (19-22). Despite molecular and cellular studies indicating that LECs have important immune functions, the contributions of these specialized cells to bacterial infections are not well understood.

Here, we show that LECs from human lymph nodes represent a niche for M. tuberculosis replication in the cytosol and autophagosomes in an RD1-dependent manner. Activation by IFN- $\gamma$ induced a cell-autonomous response, leading to bacterial growth control. We show that autophagy and the production of NO target both membrane-bound and cytosolic mycobacteria. Thus, depending on the activation state of LECs, autophagy can either promote or restrict replication. This work establishes a link between LECs and 
A

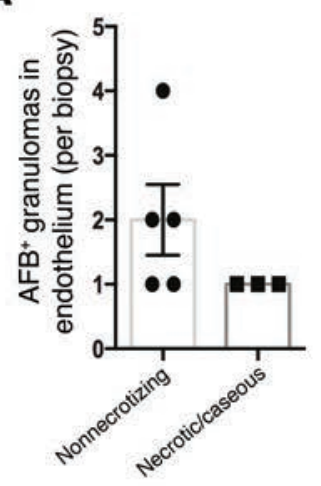

B

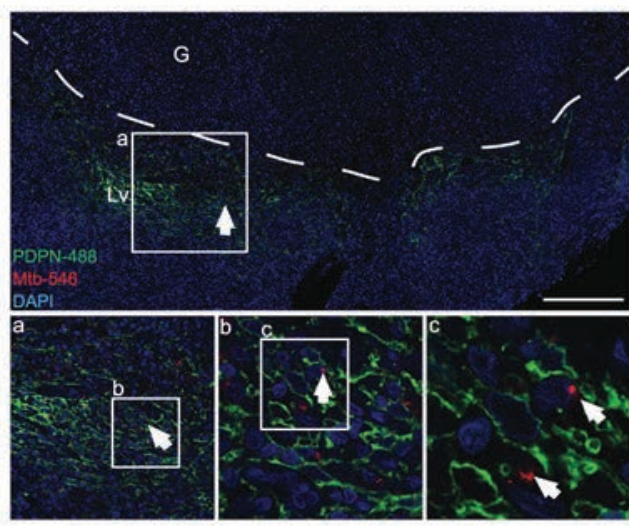

D

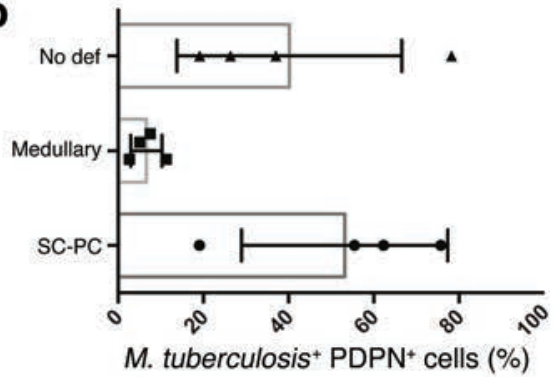

E

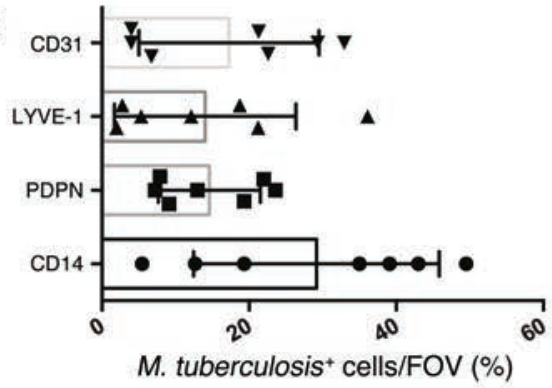

C

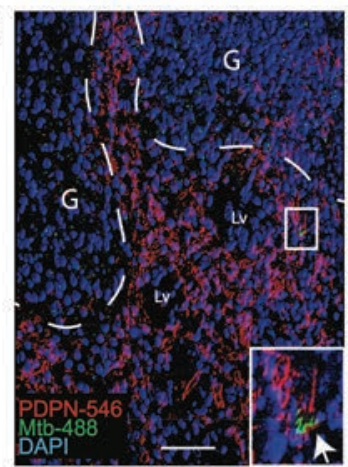

G
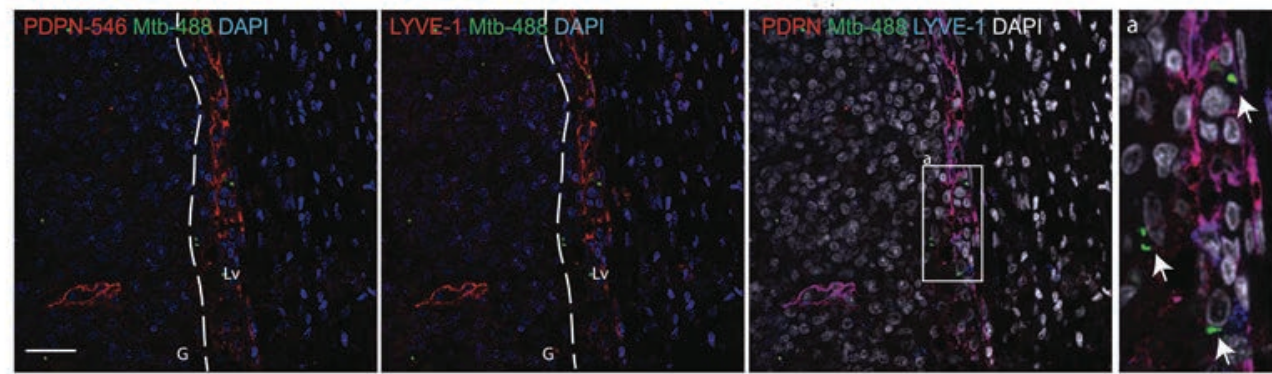

Figure 1. Primary hLECs host $\mathbf{M}$. tuberculosis. (A) The number and type of human lymph node granulomas that have AFB present within endothelial cells. Each symbol represents one biopsy. Within the 5 biopsies, there were 34 granulomas analyzed in total; 13 were positive for AFB within endothelial cells, and 21 were negative (all 21 were from 2 biopsies). (B) Representative histological sections from human patients after lymph node tissue resection surgery were stained for PDPN, M. tuberculosis, and nuclei (DAPI). Dashed line represents the border of the granuloma (G). Scale bar: $1 \mathrm{~mm}$. Original magnification, $\times 5$ (bottom left); $\times 3$ (bottom middle and right). Sequential zooms of the top image are shown. Arrows point to examples of $M$. tuberculosis-infected cells. (C) VolumeJ 3D rendering of a tissue section of a human lymph node. Two granulomas are visible, with the dashed line representing their borders. The section was stained for PDPN-Alexa Fluor 546 (PDPN-546), M. tuberculosis-Alexa Fluor 488 (Mtb-488), and nuclei (DAPI). Lv, lymphatic vessels. Scale bar: $40 \mu \mathrm{m}$. Original magnification, $\times 10$ (inset). (D) Semiquantitative analysis of the anatomical localization of the LECs positive for M. tuberculosis. SC-PC, subcapsular/paracortical; no def, not defined. (E) Quantitative analysis of the percentage of infected cells per field of view (FOV) for CD14 (myelocytic cells) and PDPN, LYVE-1, and CD31 (lymphatic and vascular endothelial cells). Seven lymph node samples were analyzed. (F) Quantitative analysis of the percentage of PDPN $+/$ LYVE-1+ cells positive for $M$. tuberculosis. (C) Representative images showing PDPN $/$ LYVE-1 $1^{+}$cells containing $M$. tuberculosis (white arrows). Scale bar: $40 \mu \mathrm{m}$. Original magnification, $\times 17$ (inset).

extrapulmonary tuberculosis and suggests that if LECs are not properly activated, they could be a reservoir for persistent infection after bacterial dissemination.

\section{Results}

Endothelial cells in lymphatics of human lymph node granulomas harbor M. tuberculosis. In granulomas from the lymph nodes of patients diagnosed with tuberculosis, we consistently found endothelial cells lining the vasculature that were infected with acid-fast bacilli ${ }^{+}$ $\left(\mathrm{AFB}^{+}\right.$), a hallmark of M. tuberculosis (Figure 1A and Supplemental Figure 1; supplemental material available online with this article;
doi:10.1172/JCI83379DS1). These podoplanin ${ }^{+}\left(\mathrm{PDPN}^{+}\right)$infected cells were mostly localized in the area surrounding the granulomas (Figure 1B) and were more frequently present in nonnecrotizing granulomas than in necrotic/caseous granulomas (Figure 1A). 3D reconstructions of the stained tissue samples (Figure 1C) showed that infected PDPN ${ }^{+}$cells were mainly localized in the subcapsular and paracortical anatomical region and were less localized in the medullary area (Figure 1D). Sections of human lymph nodes from patients with microbiological and/or histological evidence of tuberculosis were double labeled using a specific antibody for M. tuberculosis, the myelocytic marker CD14, and the endothelial 
markers PDPN, LYVE-1, or CD31 (Figure 1E). In humans, CD31 is a general marker of endothelial cells, whereas PDPN and LYVE-1 have been considered to be more specific markers of LECs (23). As expected, $29.1 \% \pm 16.7 \%$ of the $M$. tuberculosis ${ }^{+}$cells were CD $14^{+}$ per field of view, and these were primarily localized in the medullary region (data not shown). Although showing a high degree of variation across the samples, $14.6 \% \pm 6.9 \%$ of the $\mathrm{PDPN}^{+}$cells, $14.0 \% \pm 12.3 \%$ of the LYVE- $1^{+}$cells, and $17.3 \% \pm 12.2 \%$ of the CD $31^{+}$ cells were $M$. tuberculosis ${ }^{+}$(Figure 1E). When triple labeling was performed, we determined that $21.5 \% \pm 9.6 \%$ of the $\mathrm{PDPN}^{+} / \mathrm{LYVE}-$ $1^{+}$cells were $M$. tuberculosis ${ }^{+}$(Figure $1, \mathrm{~F}$ and G). In addition, we observed infected cells in the lumen of lymphatics lined by infected endothelial cells (Supplemental Figure 1). We concluded that, in human lymph nodes of patients with tuberculosis, M. tuberculosis infects various cell types, including $\mathrm{PDPN}^{+} / \mathrm{LYVE}^{-} \mathbf{1}^{+}$endothelial cells that line lymphatic vessels primarily in the subcapsular/paracortical region in association with nonnecrotizing granulomas.

$M$. tuberculosis infects human LECs in vitro, and RD1-dependent replication is restricted by $I F N-\gamma$. Next, we established an in vitro system using primary human LECs (hLECs) as an infection model. First, we confirmed that the hLECs we used for in vitro studies throughout this study were CD31 $1^{+} \mathrm{PDPN}^{+}$, and LYVE-1+ (Supplemental Figure 2A). Then, we investigated whether mycobacteria (M. tuberculosis H37Rv-EGFP [hereafter referred to as M. tuberculosis WT] and Mycobacterium bovis bacillus Calmette-GuérinEGFP [BCG-EGFP]) could infect hLECs in vitro. Using scanning electron microscopy (SEM), we observed that mycobacteria were internalized by characteristic phagocytosis-like and macropinocytosis events (Figure 2A). Since hLECs express mannose receptor (MR), which mediates mycobacterial internalization in macrophages (24), we tested whether MR has a role during internalization of M. tuberculosis into hLECs. We observed a $40 \%$ reduction of mycobacterial internalization in cells treated with mannan before infection compared with that in the untreated cells, as determined by CFU (Figure 2B). There was no significant effect in cells infected with BCG that had been pretreated with mannan prior to infection, which were used as a negative control (Figure 2B). By labeling extracellular bacteria in nonpermeabilized hLECs, we found that bacteria were internalized and not simply surface attached (Figure 2C).

We then investigated whether $M$. tuberculosis could replicate intracellularly using live-cell imaging. We followed the growth of intracellular M. tuberculosis WT for 6.5 days by live-cell imaging and determined that $M$. tuberculosis replicates intracellularly, with a doubling time of approximately 36 hours (Figure 2D and Supplemental Video 1), by plotting the EGFP signal intensity over time. The antimicrobial role of IFN- $\gamma$ against $M$. tuberculosis has been well established in macrophages, and IFN- $\gamma$ is also known to be a potent activator of hLECs (25). After confirming that hLECs were responsive to IFN- $\gamma$ in vitro, as indicated by indoleamine 2,3-dioxygenase expression and IP-10 production (Supplemental Figure 2B), we investigated the role of IFN- $\gamma$ on $M$. tuberculosis infection (Figure 2E). Confirming the results from live-cell imaging, we found that $M$. tuberculosis replicated in resting hLECs by measuring the bacterial CFU. In contrast, activated hLECs significantly restricted the bacterial growth (Figure 2E). Host cell viability was not affected, as measured by lactate dehydrogenase release assay
(Supplemental Figure 3A) or by CFU release into supernatants (Supplemental Figure 3B). In addition, the infection burden was only weakly correlated with hLEC surface area (Supplemental Figure 3C). Remarkably, M. tuberculosis grew more efficiently in hLECs than in human primary macrophages infected under comparable conditions (Supplemental Figure 3D).

In resting hLECs, M. tuberculosis tended to grow as large aggregates (Figure 2D); we therefore rationalized that our intracellular CFU values must be underestimating the total number of bacteria, since each aggregate of $M$. tuberculosis is likely to give rise to only a few CFU (data not shown). To overcome this problem, we set up an image-based system, which has been extensively used for quantifying mycobacterial growth in zebrafish $(26,27)$, to quantify $M$. tuberculosis growth using fluorescent pixels. When the sum total of all EGFP pixels per cell (raw integrated density) was determined as a measure of bacterial growth (Figure $2 \mathrm{~F}$ ), we again confirmed using this approach that $M$. tuberculosis grew in resting hLECs and IFN- $\gamma$ activation restricted this growth. We then used this method to quantify bacterial growth throughout the rest of the study. The RD1 region is important for intracellular growth of $M$. tuberculosis in epithelial cells and macrophages in vitro $(10,12)$, so we tested the role of this genomic region in hLECs (Figure 2G). M. tuberculosis $\mathrm{H} 37 \mathrm{Rv}-\triangle \mathrm{RD} 1-\mathrm{EGFP}$ (hereafter referred to as $M$. tuberculosis $\triangle \mathrm{RD} 1)$ grew more slowly and had significantly reduced growth per cell compared with $M$. tuberculosis WT in hLECs, whereas $M$. tuberculosis $\mathrm{H} 37 \mathrm{Rv}-\triangle \mathrm{RD} 1:$ RD1-EGFP (hereafter referred to as $M$. tuberculosis $\triangle \mathrm{RD} 1$ :comp) partially complemented this effect. IFN- $\gamma$ significantly restricted the growth of $M$. tuberculosis $\Delta \mathrm{RD} 1$ and $M$. tuberculosis $\triangle \mathrm{RD} 1$ :comp at 72 hours after infection. Together, these data show that in vitro hLECs can efficiently internalize mycobacteria and the MR contributes to this process. Moreover, M. tuberculosis replicates in vitro in hLECs in an RD1-dependent manner, and this growth is significantly restricted by IFN- $\gamma$.

M. tuberculosis islocalized in the cytosol and autophagosomes in an RD1-dependent manner in hLECs. In macrophages, M. tuberculosis mostly resides in an arrested phagosome lacking lysosomal markers, and this is thought to facilitate survival (28). As we observed an IFN- $\gamma$-dependent restriction of $M$. tuberculosis growth after 48 hours after infection, we analyzed the association of the bacteria at this time point with late endocytic/lysosomal markers. We used an unbiased quantitative image-based method, which facilitates measuring large numbers of mycobacteria and their association with specific cellular markers. This method circumvents the commonly used, but potentially biased, quantification strategy that utilizes visual inspection to determine a positive or negative association of bacteria with the marker. We observed a broad and heterogeneous range of associations of cathepsin D (Figure 3A) and LAMP2 (Supplemental Figure 4) with M. tuberculosis and calculated the proportion that was associated with these late endocytic markers. In resting hLECs, only $26.7 \%(n=2,272)$ (Figure $3 \mathrm{~B}$ ) and $21.6 \%$ $(n=1,029)$ (Supplemental Figure 4B) of M. tuberculosis WT were in a cathepsin $\mathrm{D}^{+}$or LAMP- $2^{+}$compartment, respectively. In contrast, $79.0 \%(n=1,414)$ of $M$. tuberculosis $\Delta \mathrm{RD} 1$ were in a cathepsin $\mathrm{D}^{+}$compartment (Figure 3B). After infection with $M$. tuberculosis $\triangle \mathrm{RD} 1$ :comp, a higher proportion of bacteria were positive for late endocytic markers compared with that after infection with $M$. tuberculosis WT, but the level was still significantly lower than that 
A

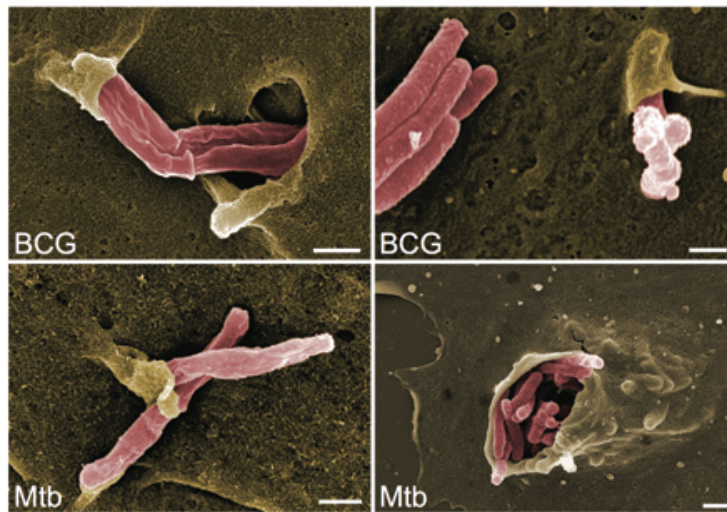

D
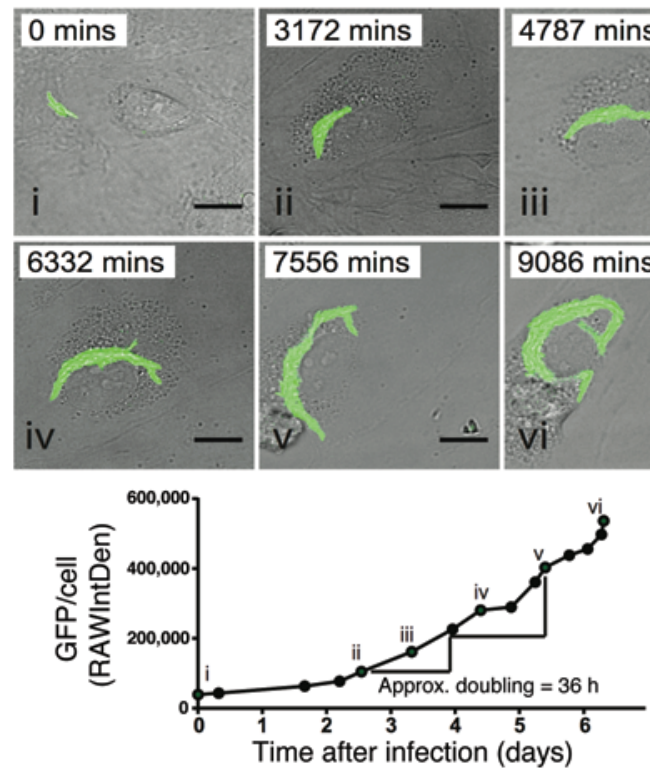

B
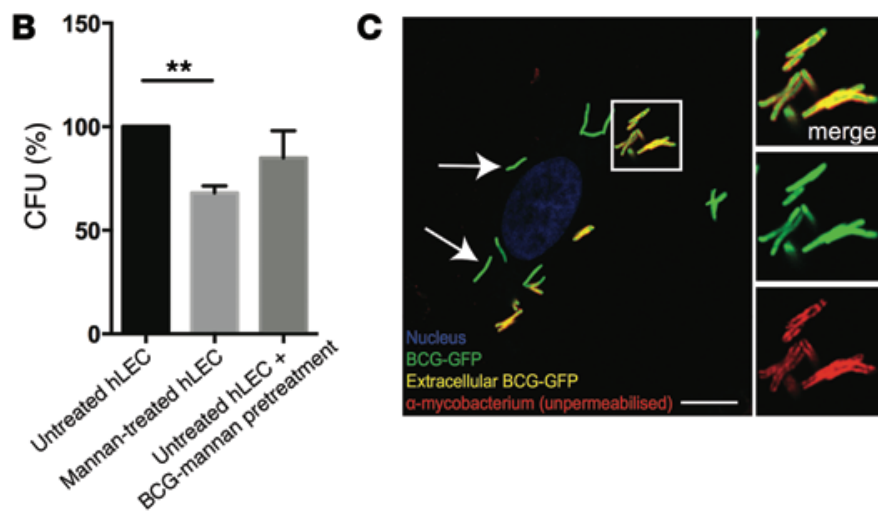

E

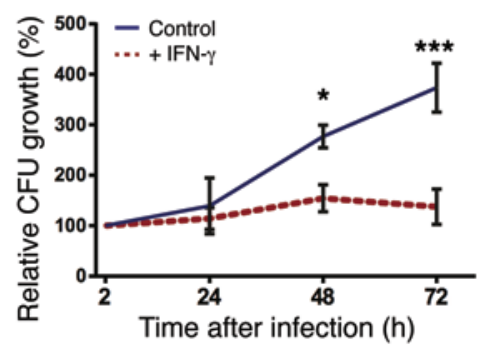

$\mathbf{F}$
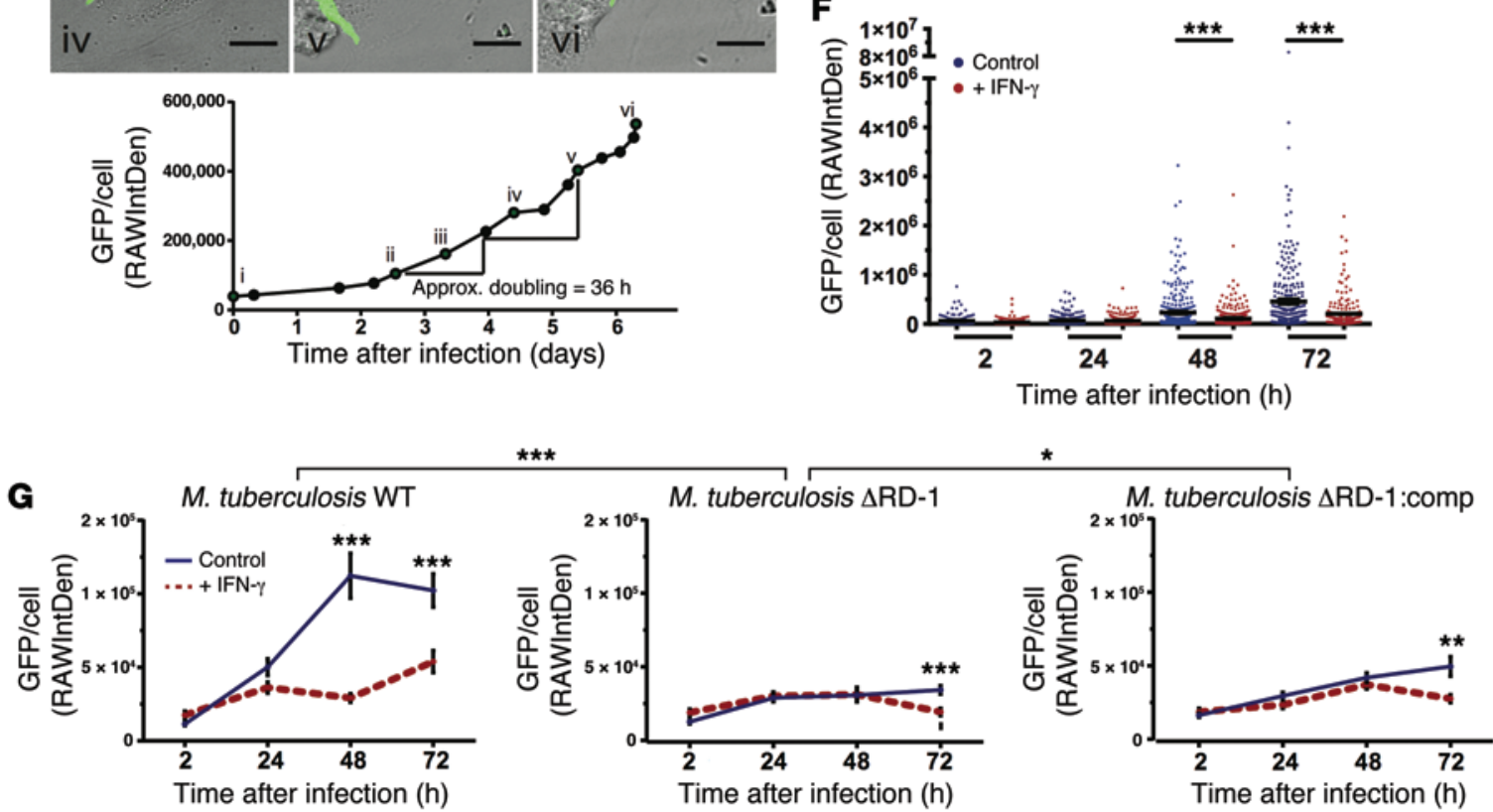

Figure 2. M. tuberculosis RD1-dependent replication in hLECs is restricted by IFN- $\gamma$. (A) SEM of hLECs after 3 hours of infection with M. bovis BCC (BCG) or M. tuberculosis WT (false-colored red). Scale bar: $500 \mathrm{~nm}$ (top row and bottom left); $1 \mu \mathrm{m}$ (bottom right). (B) Untreated hLECs were infected with BCG, and the intracellular CFU was measured after 5 hours (100\%). hLECs treated with the MR ligand mannan for 2 hours had significantly reduced uptake of BCG. BCG pretreated with mannan was used as a negative control. Error bars represent mean \pm SEM. (C) Representative image showing EGFP-BCG (white arrows, green fluorescence) and extracellular (yellow merged fluorescence) mycobacteria detected with an anti-mycobacterium antibody (red fluorescence) after 5 hours of infection. Scale bar: $10 \mu \mathrm{m}$. Original magnification, $\times 5$ (inset). (D) Stills from Supplemental Video 1. Measurement of M. tuberculosis WT growth by live-cell imaging, as shown by EGFP intensity. Scale bar: $10 \mu \mathrm{m}$. (E) Growth of M. tuberculosis WT measured by CFU at 2 to 72 hours after infection in IFN- $\gamma$-activated (red dashed line) or resting (blue line) conditions. Growth is expressed as percentage increase from the 2-hour time point. Error bars represent mean \pm SEM of 4 biological replicates. (F) Growth of $M$. tuberculosis WT inside hLECs measured by ECFP fluorescence per cell. IFN- $\gamma$-activated (red symbols) or resting (blue symbols) cells were fixed at 2, 24, 48, and 72 hours after infection. Symbols represent individual infected hLECs, and black bars represent mean \pm SEM of 4 biological replicates. (G) Growth of $M$. tuberculosis WT, M. tuberculosis $\triangle$ RD1, and M. tuberculosis $\triangle$ RD1:comp inside hLECs measured by GFP fluorescence per cell, as in F. Error bars represent mean \pm SEM of 3 biological replicates. ${ }^{*} P<0.05,{ }^{* *} P<0.01$, ${ }^{* * *} P<0.001,1$-way ANOVA with Tukey's post-hoc test. 
A M. tuberculosis WT
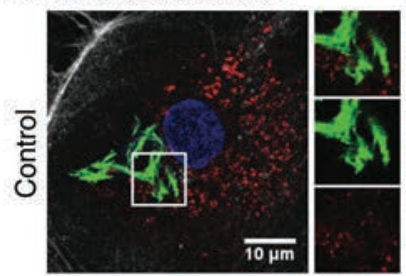

M. tuberculosis $\triangle \mathrm{RD}-1$
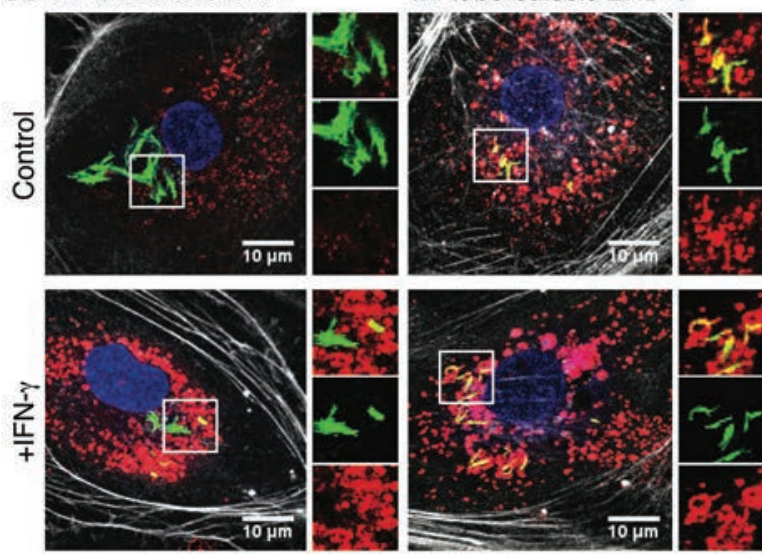

Nucleus M. tuberculosis Cathepsin D Actin
M. tuberculosis $\triangle \mathrm{RD}-1$ :comp

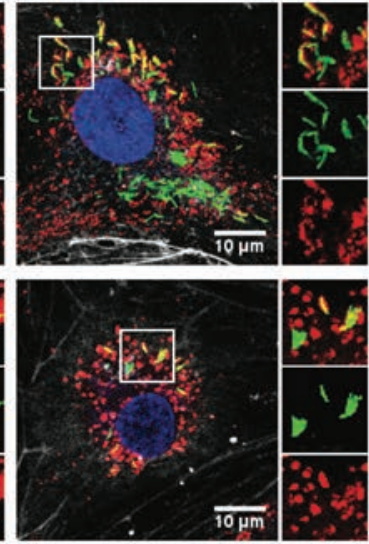

C
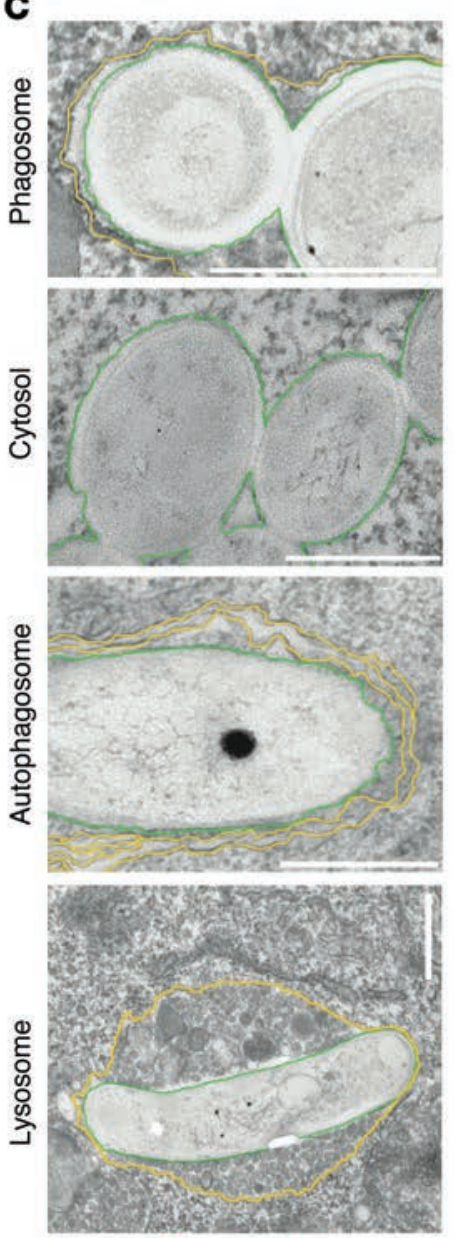

M. tuberculosis membrane Host membrane

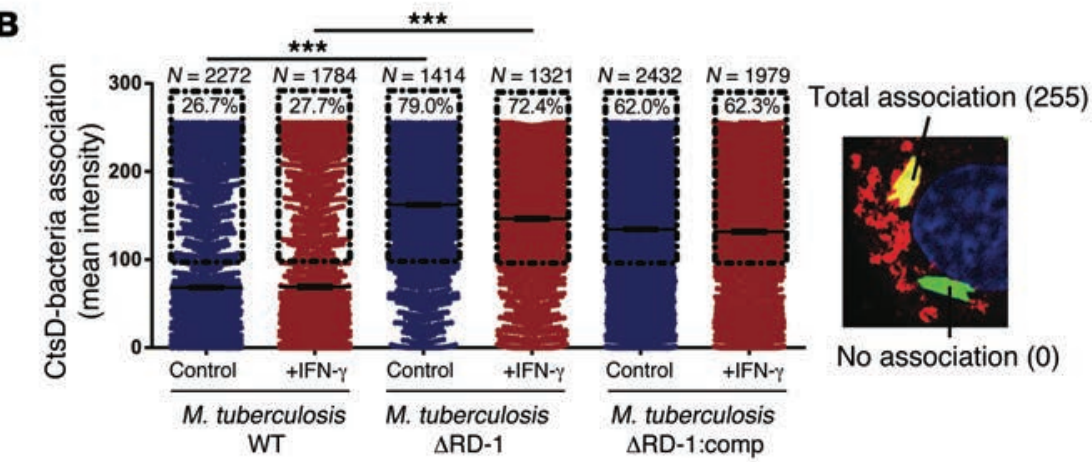

D

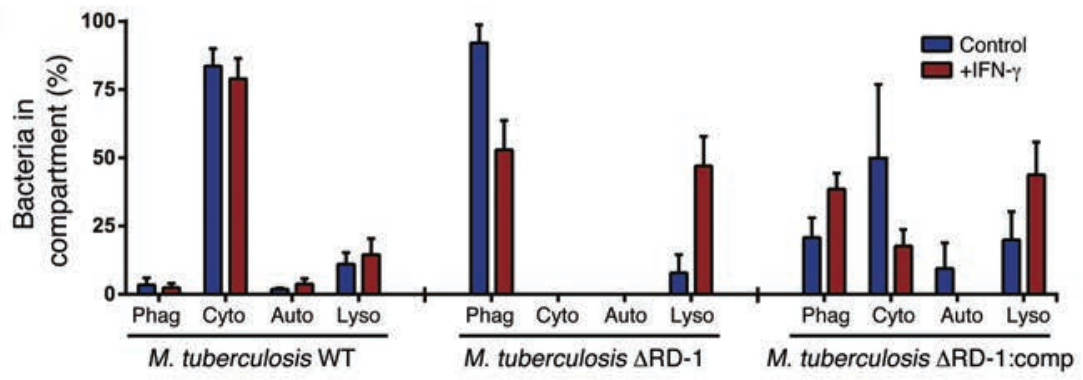

Figure 3. Subcellular localization of $\boldsymbol{M}$. tuberculosis reveals a heterogeneous localization in hLECs. (A) Representative images of hLECs infected with $M$. tuberculosis WT, M. tuberculosis $\triangle \mathrm{RD1}$, or M. tuberculosis $\triangle \mathrm{RD1}$ :comp at 48 hours after infection in the presence or absence of IFN- $\gamma$. Images show bacteria expressing EGFP, endogenous cathepsin D-Alexa Fluor 546, F-actin labeled with Alexa Fluor 633-phalloidin, and DNA labeled with DAPI. Scale bar: $10 \mu \mathrm{m}$. Original magnification, $\times 5$ (inset). (B) Quantification of the association of cathepsin D with M. tuberculosis WT, M. tuberculosis $\Delta$ RD1, or M. tuberculosis $\triangle R D 1$ :comp from images such as those in $\mathbf{A}$ at 48 hours after infection. $N$ is the total number of individual bacterial entities measured in each condition, and the percentages refer to the proportion of the cathepsin $D^{+}$population (i.e., the population within each dotted box). Error bars represent mean \pm SEM from 3 biological replicates. ${ }^{* *} P<0.001,1$-way ANOVA with Tukey's post-hoc test. (C) Example TEM images representing the observed localization of $M$. tuberculosis WT, M. tuberculosis $\triangle \mathrm{RD1}$, or $M$. tuberculosis $\triangle \mathrm{RD1}$ :comp in hLECs at 48 hours after infection. "Phagosome" represents bacteria with a single surrounding host membrane; "Cytosol" represents bacteria with no surrounding host membranes; "Autophagosome" represents bacteria found with 2 or more surrounding host membranes; and "Lysosome" represents surrounded by a host membrane filled with vesicles. Scale bar: $500 \mathrm{~nm}$. (D) Quantification of M. tuberculosis WT, M. tuberculosis $\triangle R D 1$, or M. tuberculosis $\triangle R D 1$ :comp subcellular localizations within hLECs at 48 hours after infection with in activated (red bars) or resting (blue bars) cells. Quantification was performed by stereological analysis of TEM images of resin sections of hLECs. Error bars represent mean $\pm \mathrm{SEM}$ from at least 2 biological replicates.

after infection with $M$. tuberculosis $\Delta \mathrm{RD} 1$, indicating a partial complementation of the WT strain by RD1.

We suspected that the low association of late endocytic/lysosomal markers with $M$. tuberculosis in hLECs was either because the bacteria were in arrested phagosomes or because they had translocated into the cytosol $(13,29)$. We analyzed hLECs infected with M. tuberculosis WT for 24 hours by immunogold EM labeling of LAMP-2 or CD63 on thawed Tokuyasu cryosections and observed that some bacteria did not appear to reside in a membrane-bound compartment (Supplemental Figure 5A). Using conventional resin embedding and transmission electron microscopy, we could establish that in hLECs M. tuberculosis can escape from the phagosome 
A

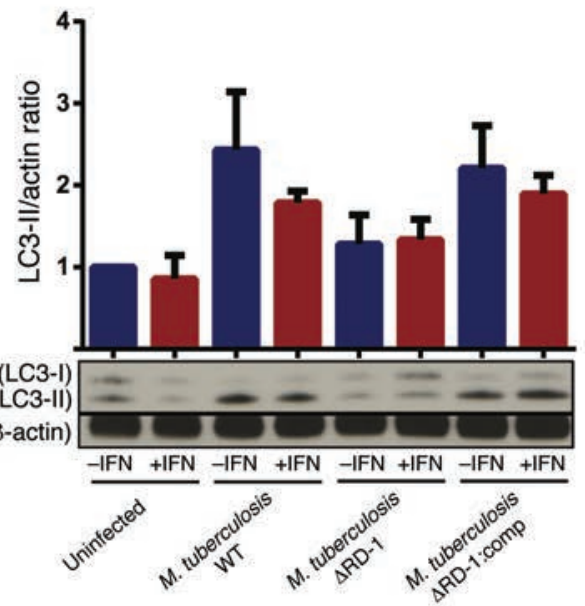

B

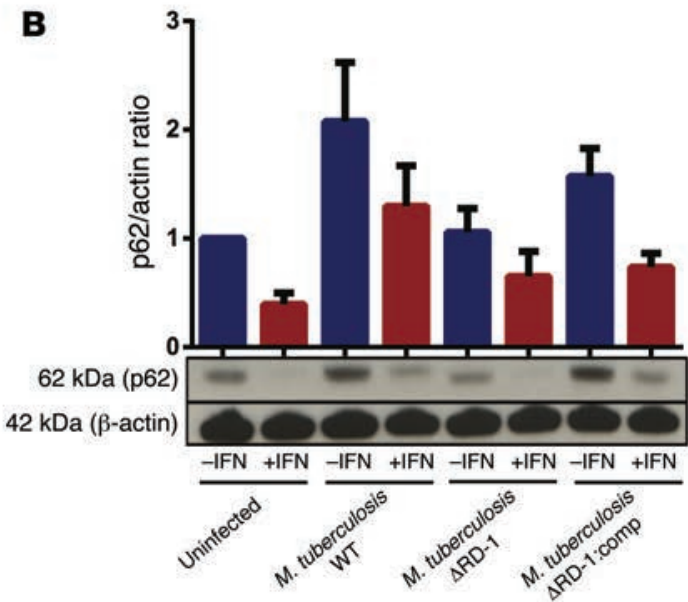

C
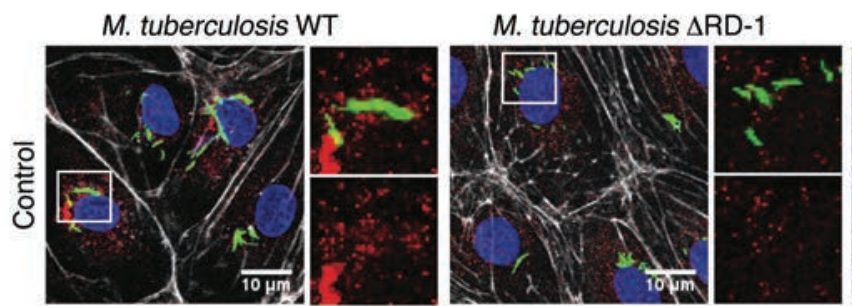

M. tuberculosis $\triangle \mathrm{RD}-1$ :comp
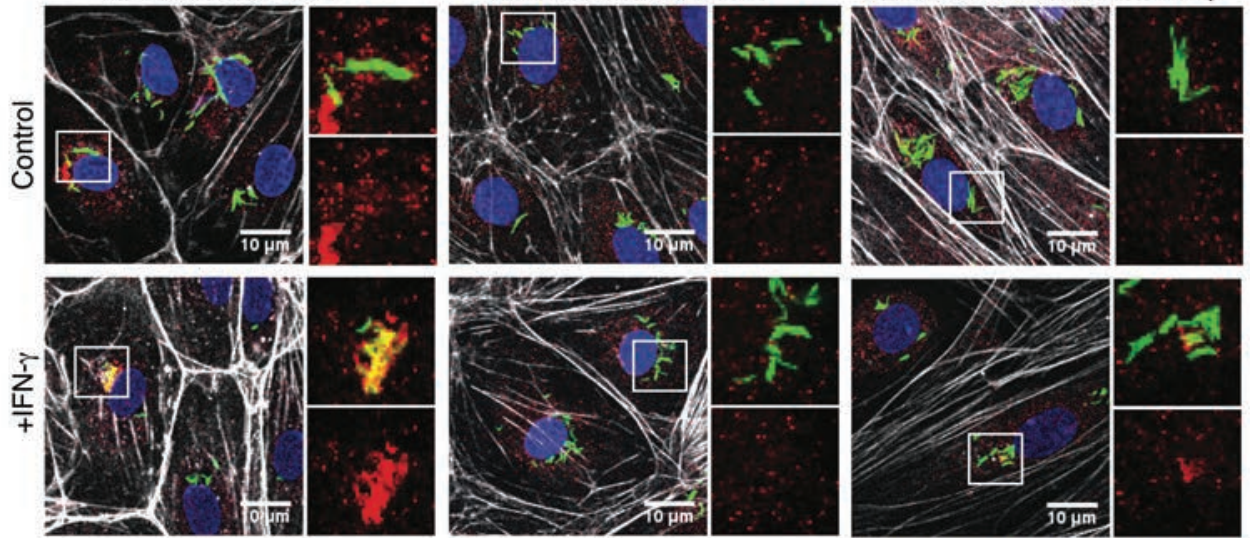

D

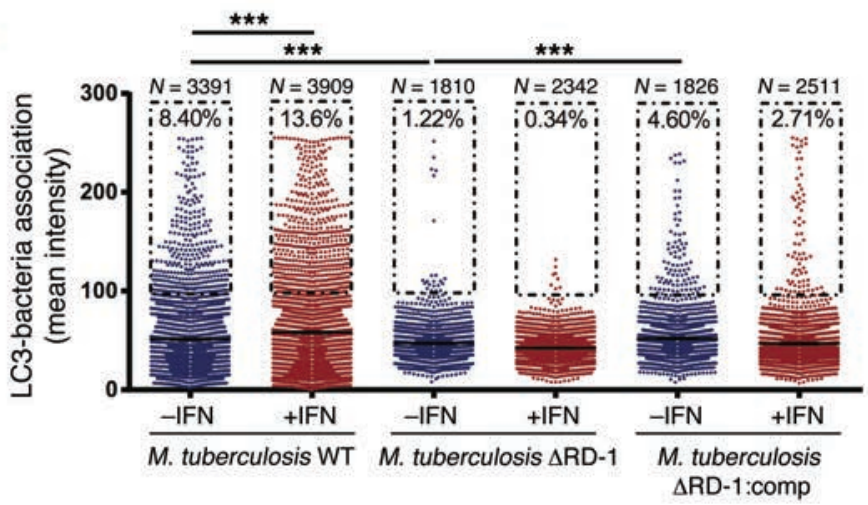

Nucleus M. tuberculosis LC3

Figure 4. Autophagy induction by M. tuberculosis and localization in autophagosomes is RD1 dependent. (A) Western blot and quantification of wholecell total protein levels of LC3-II at 48 hours after infection (normalized to actin) in uninfected, M. tuberculosis WT, M. tuberculosis $\triangle$ RD1, or M. tuberculosis $\triangle R$ RD1:comp infected hLEC samples in the presence (red bars) or absence (blue bars) of IFN- $\gamma$. Data represent mean \pm SEM of at least 3 biological replicates. (B) Same as in A, but p62 levels were measured. (C) Representative images of hLECs infected with M. tuberculosis WT, M. tuberculosis $\triangle$ RD1, or M. tuberculosis $\triangle \mathrm{RD1}$ :comp at 48 hours after infection in the presence or absence of IFN- $\gamma$. Images show bacteria expressing ECFP, endogenous LC3-Cy3, F-actin labeled with Alexa Fluor 633-phalloidin, and DNA labeled with DAPI. Scale bar: $10 \mu \mathrm{m}$. Original magnification, $\times 5$ (inset). (D) Quantification of the association of LC3 with $M$. tuberculosis WT, $M$. tuberculosis $\triangle R D 1$, or $M$. tuberculosis $\triangle R D 1$ :comp from images such as those in $\mathbf{D}$ at 48 hours after infection. $N$ is the total number of individual bacterial entities measured in each condition, and the percentages refer to the proportion of the LC $3^{+}$population (i.e., the population within each dotted box). Error bars represent mean \pm SEM from 3 biological replicates. ${ }^{* * *} P<0.001,1$-way ANOVA with Tukey's post-hoc test.

(Figure 3C and Supplemental Figure 5B). We distinguished between bacteria that were in a membrane-bound compartment (phagosomal, autophagic, or lysosomal) and those without any obvious limiting membrane in the cytosol (see Methods) (Figure 3C and Sup- plemental Figure 5B). Using stereology to calculate the percentage of bacteria in the different localizations (Figure 3D), we found that in hLECs approximately $75 \%$ of $M$. tuberculosis WT were present in the cytosol at 48 hours after infection, while $M$. tuberculosis $\triangle \mathrm{RD} 1$ 
remained entirely in membrane-bound compartments. The cytosolic fraction of $M$. tuberculosis $\Delta \mathrm{RD} 1$ :comp was $50 \%$, indicating that complementation was functional with regard to cytosolic localization. Importantly, treatment with IFN- $\gamma$ did not change the localization pattern of $M$. tuberculosis WT considerably, although the $M$. tuberculosis $\triangle \mathrm{RD} 1$ was more likely to reside in late endosomal/lysosomal compartments at 48 hours after infection. Altogether, only a minor fraction of M. tuberculosis WT localized in late endocytic compartments, either in resting or IFN- $\gamma$-activated cells. Conversely, the M. tuberculosis $\triangle \mathrm{RD} 1$ mutant was mostly localized in phagolysosomes in both conditions. We concluded that in hLECs M. tuberculosis is localized in the cytosol by 48 hours after infection in a RD1dependent manner. Moreover, the presence of $M$. tuberculosis WT in autophagosomes was RD1 dependent, since $M$. tuberculosis $\triangle \mathrm{RD} 1$ were never found in these organelles.

Autophagy induction by M. tuberculosis and localization in autophagosomes is RD1 dependent. Given that autophagy is an IFN- $\gamma$ mediated mechanism that controls intracellular mycobacteria in macrophages (30) and that cytosolic mycobacteria can be targeted to autophagosomes (31), we investigated whether this pathway was induced in hLECs. The number of LC3 puncta was significantly higher after IFN- $\gamma$ stimulation at 48 hours (Supplemental Figure $6 \mathrm{~A})$, arguing for induction of autophagy and formation of autophagosomes. Infection of hLECs with $M$. tuberculosis WT led to an accumulation of LC3-II in an RD1-dependent manner, although the total levels were similar in resting and activated cells (Figure $4 \mathrm{~A})$. We expected that the LC3-II levels would be higher in IFN- $\gamma$ treated cells, but LC3-II itself is degraded by autophagy, which could mask this effect. Complementation of the RD1 region mostly restored the LC3-II accumulation. Similarly, the autophagy adaptor protein $\mathrm{p} 62$ accumulated after infection with $M$. tuberculosis WT in an RD1-dependent manner (Figure 4B). The total protein levels of p62 were reduced by approximately $30 \%$ to $40 \%$ after IFN- $\gamma$ treatment (Supplemental Figure 6B), consistent with autophagyinduced degradation of p62 (32). By analyzing the association of LC3 with $M$. tuberculosis by confocal microscopy (Figure 4, C and D), we confirmed that autophagic targeting is strictly RD1 dependent. At 48 hours after infection, the association of LC3 with M. tuberculosis WT in resting hLECs was relatively low $(8.4 \%$, $n=3,391)$, while IFN- $\gamma$ treatment significantly increased the association of LC3 with bacteria $(13.6 \%, n=3,309)$ (Figure $4 \mathrm{D}$ ) in agreement with the EM results (Figure 3D). As expected, the association of LC3 with $M$. tuberculosis $\triangle \mathrm{RD} 1$ was almost absent $(1.22 \%, n=1,810)$, while the association of LC3 with M. tuberculosis $\triangle \mathrm{RD} 1$ :comp was slightly elevated compared with that of the M. tuberculosis $\Delta \mathrm{RD} 1$ strain $(4.6 \%, n=1,826)$, consistent with a partial, although functional, complementation of the RD1 region.

Autophagy promotes $M$. tuberculosis growth in resting $h L E C s$ but restricts growth in activated $h L E C s$. To investigate the effect that autophagy has on the intracellular growth of M. tuberculosis, we infected hLECs with $M$. tuberculosis WT, M. tuberculosis $\triangle \mathrm{RD} 1$, and $M$. tuberculosis $\Delta \mathrm{RD} 1$ :comp for 48 hours, in the presence or absence of the autophagy inhibitor 3-methyl adenine (3-MA) and IFN- $\gamma$. The EGFP intensity per hLEC was quantified for each condition as a measure of $M$. tuberculosis growth (Figure 5A). In IFN- $\gamma-$ activated cells, 3-MA overcame the IFN- $\gamma$-dependent restriction of $M$. tuberculosis WT growth (Figure 5A), suggesting that activated
hLECs use autophagy as a mechanism to control bacterial growth. As expected, this effect was not observed for M. tuberculosis $\triangle \mathrm{RD} 1$, which could not proliferate in hLECs (Figure 2G). In contrast to the effect observed in activated hLECs, in resting conditions 3-MA reduced the growth of $M$. tuberculosis WT, M. tuberculosis $\triangle \mathrm{RD} 1$, and M. tuberculosis $\Delta \mathrm{RD} 1$ :comp by $25.9 \%, 36.8 \%$, and $52.4 \%$, respectively (Figure $5 \mathrm{~A}$ ). This argues that in resting cells autophagy may actually promote bacterial growth rather than restrict it. To investigate this hypothesis further, we inhibited autophagy using siRNA to knockdown (KD) the essential autophagy protein ATG5 (Supplemental Figure 6C). We then infected control hLECs or ATG5 KD hLECs with M. tuberculosis WT for 48 hours in resting and activating conditions (Figure $5 \mathrm{~B}$ ). In resting conditions, ATG5 $\mathrm{KD}$ resulted in significantly fewer bacteria per cell, indicating that autophagy is beneficial to M. tuberculosis growth. Conversely, in activated hLECs, ATG5 KD resulted in significantly more bacteria per cell, indicating that activation of hLECs by IFN- $\gamma$ restricts $M$. tuberculosis growth. Moreover, in resting conditions, induction of autophagy by rapamycin also increased bacterial growth (Figure $5 \mathrm{C}$ ). Thus, under resting conditions, autophagy is beneficial to M. tuberculosis, as there were significantly more bacteria per hLEC under autophagy-promoting conditions.

We hypothesized that $M$. tuberculosis was growing in $\mathrm{LC}^{+}$ autophagic compartments in resting cells. To test this, we developed a correlative light and electron microscopy (CLEM) approach, incorporating live-cell imaging that allowed us to determine the relationship between $\mathrm{LC}^{+}$compartments at the ultrastructural level with the growth of live bacteria. We found that $M$. tuberculosis within LC3-RFP compartments were actively growing in resting hLECs (Figure 5D). Bacteria growing over a 5-day period were localized in an ultrastructurally very complex LC3$\mathrm{RFP}^{+}$compartment containing multiple membranes and vesicles (Figure 5D and Supplemental Videos 2 and 3). We concluded that autophagy can both promote and restrict bacterial growth, depending on the state of activation.

$N O$ production restricts the growth of $M$. tuberculosis in activated hLECs. The most rapid period of M. tuberculosis growth in hLECs corresponded with its shift to a cytosolic localization (Figure $2 \mathrm{E}$ and Figure 3D). Although autophagic targeting can restrict bacterial growth (Figure 5, A-C), the subpopulation of M. tuberculosis targeted to autophagosomes at any time was relatively small (Figure 4D). Thus, we investigated the possibility that another IFN- $\gamma-$ dependent host mechanism might operate in the cytosol to control bacterial growth. An alternative bacterial-killing mechanism to consider was the generation of NO by the host. LECs express both the inducible NOS (iNOS) and the endothelial NOS (eNOS) (19). Using the marker DAF-FM diacetate, which becomes fluorescent upon reaction with NO, we detected increased levels of intracellular NO in hLECs after IFN- $\gamma$ activation, and the effect was blocked using the NOS inhibitor L-NG-monomethyl arginine citrate (L-NMMA) (Figure 6A). The increase in NO production was not due to changes in enzyme expression, since the iNOS and eNOS levels remained similar, both after IFN- $\gamma$ activation or infection (Figure 6B). We did not observe any IFN- $\gamma$-dependent effects on phosphorylation of eNOS (data not shown). Crucially, we observed that eNOS colocalized with $M$. tuberculosis in IFN- $\gamma$-activated cells 3 times more frequently than in resting cells (Figure 6, C and D). 

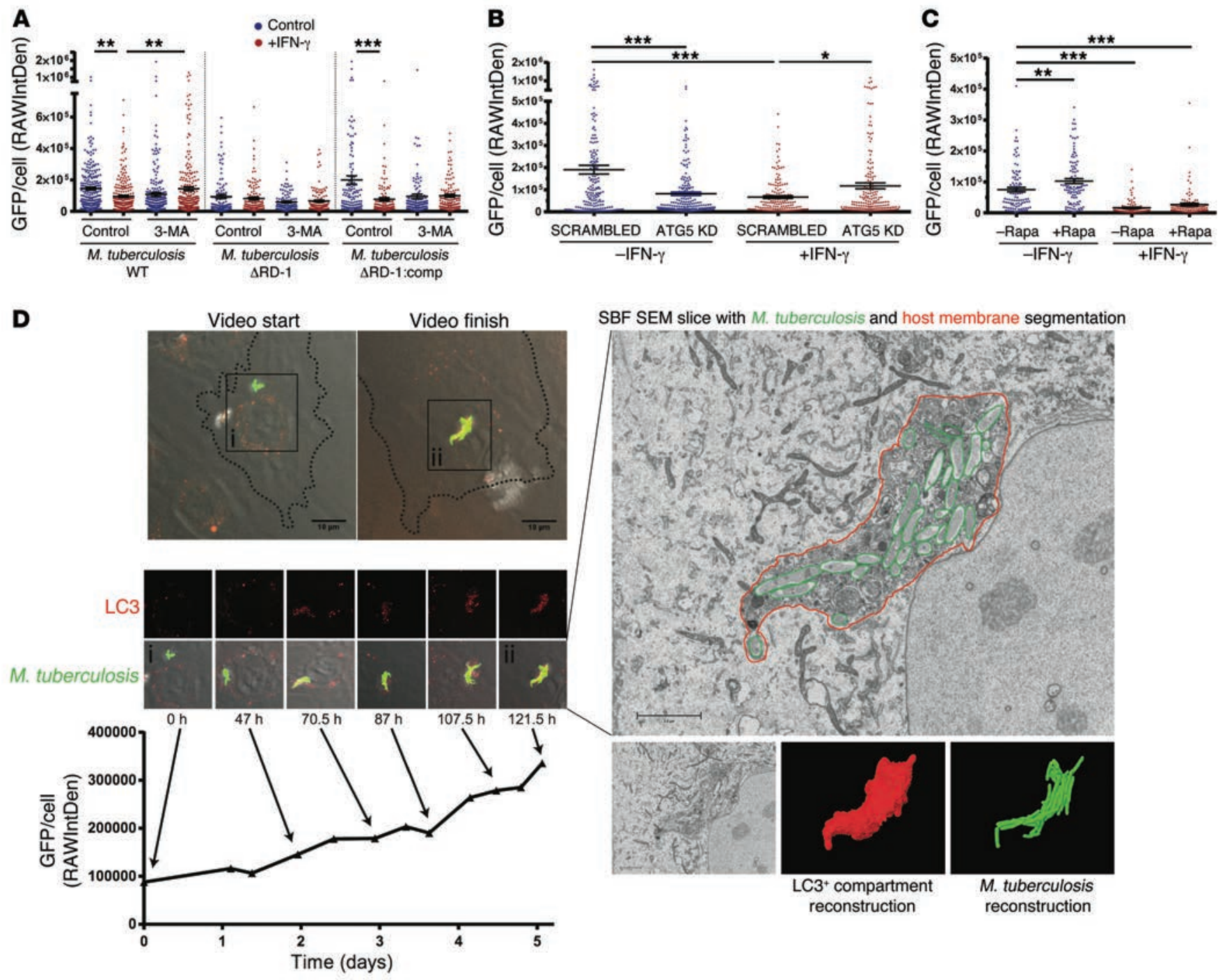

SBF SEM slice with $M$. tuberculosis and host membrane segmentation

Figure 5. Autophagy promotes M. tuberculosis growth in resting hLEC. (A) The growth (GFP fluorescence per cell) of M. tuberculosis WT, M. tuberculosis $\triangle \mathrm{RD1}$, or M. tuberculosis $\triangle \mathrm{RD1}$ :comp after 48 hours of infection in hLECs treated or not (control) with $10 \mathrm{mM} 3-\mathrm{MA}$ in activated (red symbols) or resting (blue symbols) cells. Error bars represent mean \pm SEM of at least 3 biological replicates. (B) The growth (EGFP fluorescence per cell) of $M$. tuberculosis WT after 48 hours of infection in control cells (SCRAMBLED) or cells with reduced ATG5 protein level (ATG5 KD) in resting (blue symbols) or activating (red symbols) conditions. Error bars represent mean \pm SEM of 3 biological replicates. (C) The growth (EGFP fluorescence per cell) of $M$. tuberculosis WT after 48 hours of infection in control cells (-Rapa) or cells treated with $500 \mathrm{nM}$ rapamycin (+Rapa) in resting (blue symbols) or activating (red symbols) conditions. Error bars represent mean \pm SEM of 3 biological replicates. NS $=P>0.05 ;{ }^{*} P<0.05$, ${ }^{*} P<0.01,{ }^{* * *} P<0.001$, 1-way ANOVA with Tukey's post-hoc test. (D) Images taken from live-cell imaging of LC3-RFP-expressing hLECs infected with $M$. tuberculosis WT over 5 days in resting conditions. Scale bar: $10 \mu \mathrm{m}$. GFP fluorescence was plotted to show growth of the bacteria in an $\mathrm{LC3}^{+}$compartment. The structure was relocated for serial block-face SEM analysis using a correlative workflow, and the serial electron images were manually segmented and rendered to create a 3D model of the bacteria (green) and the limiting membrane (red). Scale bar: $2.5 \mu \mathrm{m}$. See also Supplemental Videos 2 and 3.

Colocalization was only observed at background levels with iNOS in activated or resting cells (Figure 6, C and D). This suggests that IFN- $\gamma$ can facilitate the targeting of existing pools of intracellular eNOS to M. tuberculosis. Importantly, the inhibition of NO production by L-NMMA abolished the effect of IFN- $\gamma$ in the restriction of $M$. tuberculosis growth at 48 hours after infection, whereas this effect was not observed in cells infected with $M$. tuberculosis $\triangle \mathrm{RD} 1$ (Figure 6E). In addition, we observed that treatment with L-NMMA inhibited the growth of the strains that had a high proportion of membrane-bound bacteria, suggesting that NOS may affect the membrane-bound populations in a different way. We concluded that NO production is one of the factors that restrict the growth of M. tuberculosis WT. The fact that we observed that eNOS ${ }^{+} / \mathrm{PDPN}^{+}$ cells are positive for M. tuberculosis in human lymph node samples (Figure 6F) supports the role of this enzyme in the control of human extrapulmonary tuberculosis.

\section{Discussion}

In this study, we identified LECs as a natural niche for M. tuberculosis replication in human lymph nodes. This opens the possibility that the lymphatic endothelium and LECs are more active players in the development of lymphatic tuberculosis than previously con- 

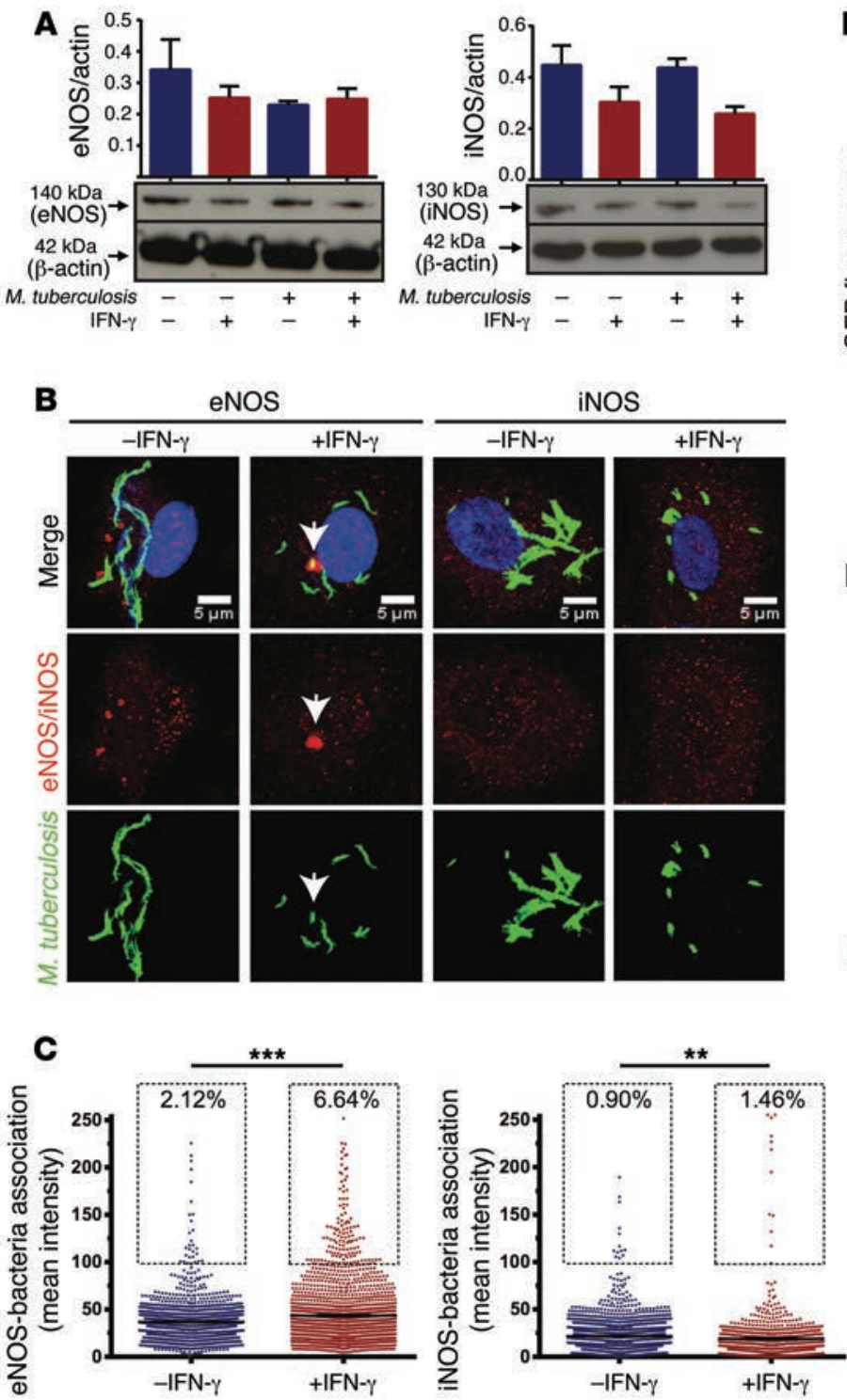

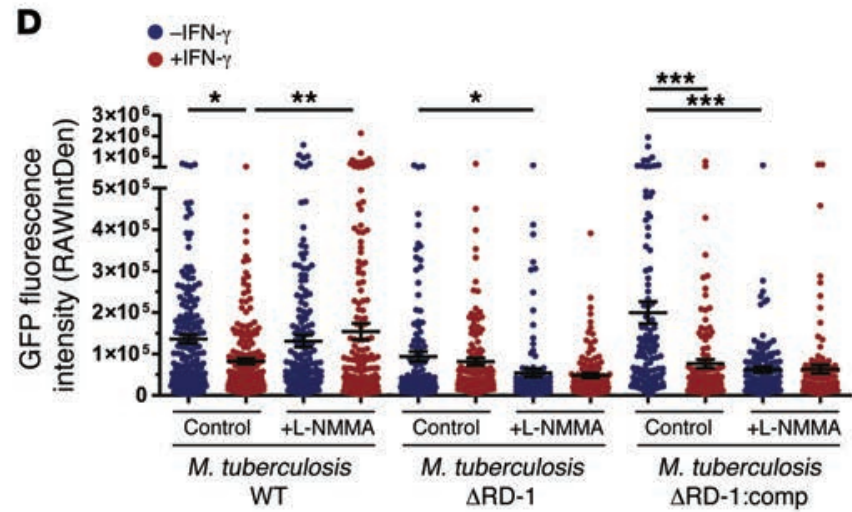

E

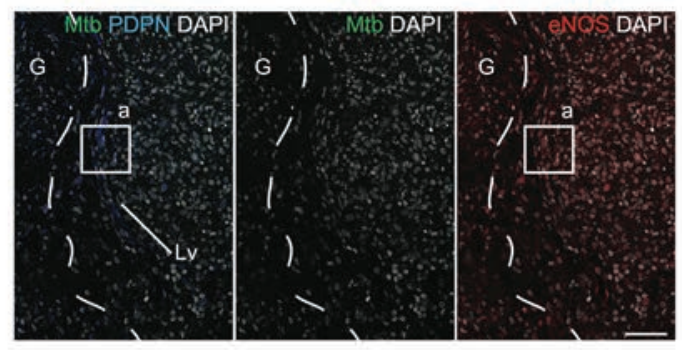

$\mathbf{F}$
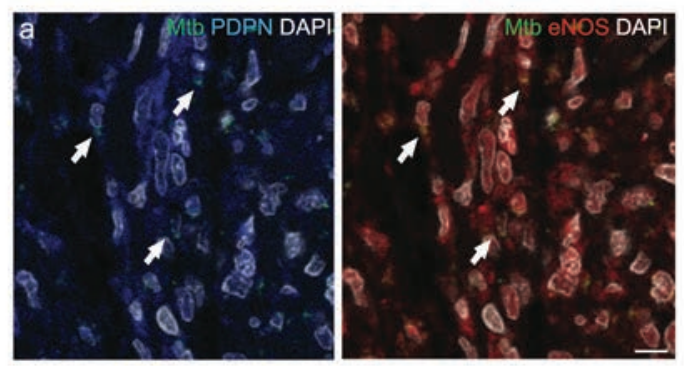

Figure 6. NO production restricts the growth of cytosolic $\mathbf{M}$. tuberculosis. (A) Western blot and quantification of the whole-cell protein levels of eNOS and iNOS at 48 hours after infection (normalized to actin) in uninfected and $M$. tuberculosis WT-infected activated (red bars) or resting (blue bars) hLEC samples. Error bars represent mean \pm SEM of 4 biological replicates. (B) Images showing representative areas of $M$. tuberculosis WT-infected hLECs after 48 hours of infection in activated or resting conditions. Images show bacteria expressing EGFP, endogenous eNOS- or iNOS-Alexa Fluor 546, and DNA labeled with DAPI. Arrows show bacteria and eNOS colocalization. Scale bar: $5 \mu \mathrm{m}$. (C) Quantification of the association of eNOS (top panel) and iNOS (bottom panel) with $M$. tuberculosis WT from images such as those in $\mathbf{C}$ at 48 hours after infection. The percentages refers to the proportion of the eNOS ${ }^{+} /$iNOS $^{+}$population (i.e., the population within each dotted box). Error bars represent mean \pm SEM from at least 4 biological replicates. ${ }^{* *} P<0.01,{ }^{* *} P<0.001,2$-tailed Student's $t$ test. (D) The growth (GFP fluorescence per cell) of M. tuberculosis WT, M. tuberculosis $\Delta$ RD1, or M. tuberculosis $\triangle \mathrm{RD1}$ :comp after 48 hours of infection in untreated (control) hLECs or hLECs treated with $250 \mu \mathrm{M}$-NMMA in activated (red symbols) or resting (blue symbols) conditions. Error bars represent mean \pm SEM of at least 3 biological replicates. ${ }^{*} P<0.05$, ${ }^{* *} P<0.01$, ${ }^{* * *} P<0.001,1$-way ANOVA with Tukey's post-hoc test. (E) Representative histological section of human lymph nodes stained for PDPN, M. tuberculosis, eNOS, and nuclei (DAPI). The dotted line indicates the granulomatous area. Scale bar: $50 \mu \mathrm{m}$. (F) Images of the lymphatic vessel region showing localization of $M$. tuberculosis in $\mathrm{PDPN}^{+}$and eNOS+ cells (white arrows). Scale bar: $10 \mu \mathrm{m}$.

sidered. The association of the lymphatic endothelial network with M. tuberculosis granulomas has previously been shown in the lymph nodes of nonhuman primates (33) and the lungs of guinea pigs (34). We provide clinical evidence that hLECs harbor M. tuberculosis in lymphatic vessels associated with granulomas in lymph nodes. Consistent with this idea, it has recently been shown that tuberculous granulomas express VEGF and mycobacterial infection induc- es lymphatic remodeling and lymphangiogenesis via the expression of VEGFR-3 $(35,36)$. Although DNA from $M$. tuberculosis has been found in the endothelium of human lungs (16), whether LECs lining the lymphatic system in the lung can also be infected with $M$. tuberculosis is an open question. Myelocytic cells can express endothelial markers, and they can be the precursors of new lymphatics (37). In our studies, the morphological structure and the colocal- 


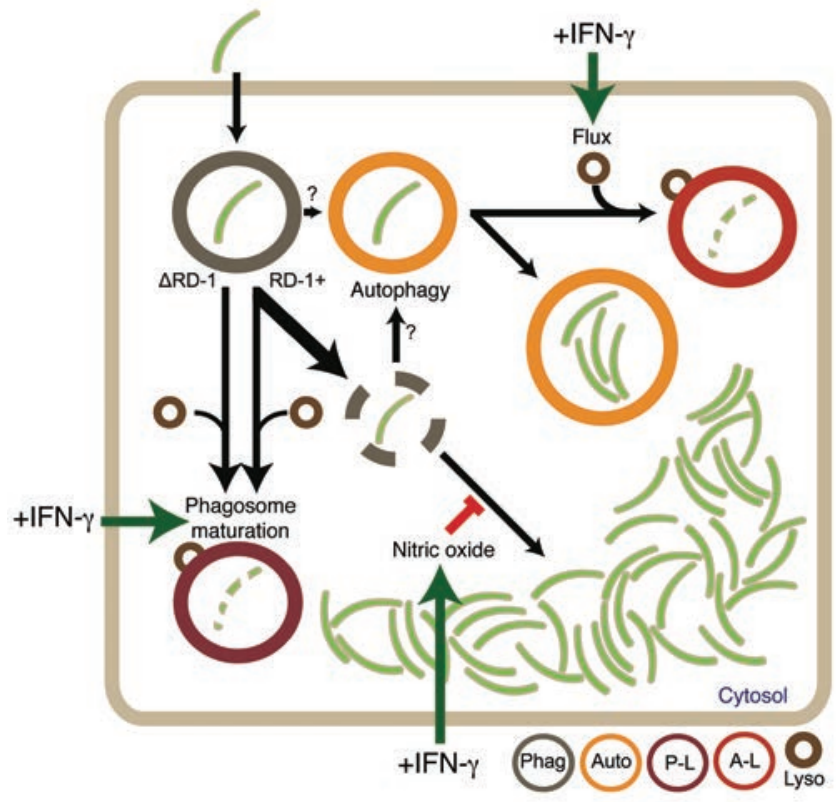

ization in the lymph node, as shown by PDPN and LYVE-1 labeling, are consistent with that in lymphatic vessels. We cannot exclude that newly recruited myelocytic cells contributing to the inflammatory lymphangiogenic process could also harbor bacteria. In this context, the idea that $M$. tuberculosis-infected myelocytic precursors may contribute to lymphangiogenesis in the granuloma is an interesting hypothesis that warrants further investigation.

hLECs express receptors important for the response to pathogens, including the MR $(18,38)$. Together with the complement receptors, MRs are the most common pathways by which mycobacteria are internalized into macrophages (24). We show that mycobacteria also use MR to be internalized in hLECs, and our SEM studies reinforce the idea that LECs are indeed capable of phagocytosis (19). Moreover, we found that M. tuberculosis actively grows in LECs after internalization. However, our results in LECs show considerable differences compared with myelocytic cells in the localization of M. tuberculosis, with important functional consequences. First, the fraction of cytosolic $M$. tuberculosis in infected hLECs is much higher than it is in myelocytic cells (29). Second, in hLECs, bacterial translocation into the cytosol occurred without compromising cell viability, in contrast to macrophages in which necrotic cell death is observed (15). Thus, M. tuberculosis may be translocated to the cytoplasm faster and more frequently in resting hLECs than in myelocytic cells, because the hLEC cytosol could represent a more permissive environment for $M$. tuberculosis growth. In support of this hypothesis, we observed that (a) in vitro $M$. tuberculosis replicates better in hLECs than in primary human macrophages and (b) M. tuberculosis lacking the RD1 region was not able to localize to the cytosol of hLECs, and its proliferation was severely impaired. Even in resting cells, the M. tuberculosis $\triangle \mathrm{RD} 1$ strain colocalized much more frequently with markers for late endosomes/lysosomes than $M$. tuberculosis WT, suggesting that in hLECs escaping into the cytosol is a more effective survival strategy than blocking phagosome maturation.

IFN- $\gamma$ is known to be a major factor in the interaction of M. tuberculosis with macrophages and in granuloma formation.
Figure 7. Proposed model of $M$. tuberculosis dynamics in infected hLECs. M. tuberculosis (green) are phagocytosed by hLECs and initially reside in the phagosome (brown). At 24 hours after infection, $70 \%$ to $90 \%$ of the bacteria escape into the cytosol, but this is strictly RD1 dependent. M. tuberculosis lacking RD1 and a small fraction of $\mathrm{RD}^{+} \mathrm{M}$. tuberculosis are trafficked directly to phagolysosomes (P-L) and suffer restricted growth/killing (this process is also induced by IFN- $\gamma$ ). In the cytosol, $M$. tuberculosis can readily replicate, but this is restricted when IFN- $\gamma$ is present via the relocalization of intracellular eNOS to generate NO in the immediate vicinity of the bacteria. A small population of $M$. tuberculosis also enters the autophagy pathway in an RD1-dependent manner, directly from the phagosome and/or via the cytosol. M. tuberculosis induces autophagosome formation and can replicate in an $\mathrm{LC}^{+}$compartment by blocking fusion of lysosomes with the autophagosome. Upon activation of hLECs by IFN- $\gamma$, autophagosomelysosome fusion is restored (i.e., increased autophagic flux), and M. tuberculosis growth is restricted in autophagolysosomes (A-L).

The effect of IFN- $\gamma$ has been extensively characterized in macrophages, since these cells have long been known to harbor M. tuberculosis in vivo (39). However, nonmyelocytic cells are also required for tuberculosis control in a mouse model (7). Our data indicate that, in addition to myelocytic cells, hLECs may also support the growth of $M$. tuberculosis, and IFN- $\gamma$ activates a cell-autonomous antibacterial response in these cells. IFN- $\gamma$ was strikingly efficient in restricting $M$. tuberculosis growth in hLECs, yet the bacteria remained primarily cytosolic, leading to the question of how this cytokine works in these cells. Our data argue that in hLECs IFN- $\gamma$ triggers at least two mechanisms that restrict the growth of mycobacteria: one that targets membrane-bound bacteria and another that targets cytosolic bacteria. The effect of IFN- $\gamma$ promoting phagosome maturation was relatively modest, suggesting that this is not the primary mechanism of growth restriction. In contrast, the targeting of the mycobacteria to $\mathrm{LC}^{+}$compartments in hLECs was significantly affected by IFN- $\gamma$, although only in a subpopulation of the bacteria, as previously reported in mouse macrophages (14).

The RD1-dependent accumulation in hLECs of LC3 and p62 after infection indicates that autophagy is induced and autophagosomes are formed but autophagic degradation (flux) is blocked, likely due to defective fusion of autophagosomes with lysosomes. In this scenario, RD1-mediated secretion of bacterial components could block autophagic flux. Alternatively, translocation of $M$. tuberculosis into the cytosol could be a prerequisite for subsequent recapture into the autophagic pathway, in which case M. tuberculosis-encoded factors could be directly secreted into the host cytosol. Interestingly, IFN- $\gamma$ activation leads to a reduction in p62 levels, indicative of restored autophagic flux, and thus contributes to the restriction of $M$. tuberculosis growth.

In line with these results, our CLEM analysis revealed that a subpopulation of $M$. tuberculosis is able to grow in $\mathrm{LC}^{+}$compartments surrounded by $\mathrm{LC}^{+}$vesicles in resting hLECs. Although many pathogens replicate in autophagic compartments, $M$. tuberculosis replication in autophagosomes was unexpected, considering the established role of autophagy in the antimycobacterial 
response in myelocytic cells (30). However, our results provide an explanation for the observation that clinical isolates of $M$. tuberculosis manipulate autophagy for their survival (40) and mirror those seen in M.tuberculosis infection of human primary dendritic cells (41), human type II alveolar epithelial cells (42), and some primary alveolar macrophages from adult macaques (43). Consistent with our observations, in Listeria-infected macrophages, bacteria are localized to both the cytosol and $\mathrm{LC}^{+}$compartments in which bacteria grow (44), and long-term infection of macrophages with $M$. bovis BCG also leads to growth of the bacteria in $\mathrm{LC}^{+}$compartments (45).

In resting hLECs, there was a 3-MA-sensitive fraction of M. tuberculosis that proliferated, in addition to a large pool of cytosolic M. tuberculosis that was 3-MA insensitive. In activated cells, 3-MA treatment reversed the IFN- $\gamma$ dependent growth restriction. The effects of IFN- $\gamma$ were RD1 dependent, since $M$. tuberculosis $\triangle \mathrm{RD} 1$ bacteria were not found in autophagosomes; rather, this strain resided in phagosomes that rapidly acquired late endosomal markers. Together with our ATG5 KD and rapamycin studies, we confirmed that, in resting hLECs, blocking the autophagy pathway leads to $M$. tuberculosis growth restriction, whereas inducing this pathway results in $M$. tuberculosis growth.

Having identified a large pool of cytosolic M. tuberculosis in hLECs, our results indicate a role for NO in the autonomous response of hLECs to $M$. tuberculosis. In addition to its effects on autophagy, we found that IFN- $\gamma$-dependent NO production was required to control bacteria replicating in the cytosol after 48 hours of infection. In hLECs, there are two NOSs: iNOS and eNOS. The role of iNOS is well recognized in the context of phagocyte activation, particularly in mice. The intracellular distribution of eNOS is an important mechanism for regulating the spatiotemporal production of NO (47). In infected hLECs, the regulation of NO is independent of enzyme expression, since both iNOS and eNOS are constitutively expressed during bacterial growth. We found a correlation between the intracellular location of eNOS and the restriction of bacterial growth in response to IFN- $\gamma$. Although the NO levels produced by eNOS are lower than those produced by iNOS (46), the cytosolic fraction of the enzyme could be sufficient to generate short-lived NO precisely where the cytosolic bacteria replicate. Supporting this idea, we observed $M$. tuberculosis localization in $\mathrm{PDPN}^{+} / \mathrm{eNOS}^{+}$cells from human tissue samples. eNOS is upregulated in the acute phase of experimental bacterial meningitis (47). In macrophages, eNOS contributes to the killing of Streptococcus pneumoniae (48). Supporting an important role for this enzyme in tuberculosis, eNOS is expressed and functional in the granulomas of macaques, and other isoforms different from iNOS contribute to NO production in infected tissues (49).

Collectively, our data reveal a scenario whereby, in hLECs, in addition to a large pool of $M$. tuberculosis growing in the cytosol, there is a subpopulation of bacteria that replicate in autophagosomes (Figure 7). This model highlights the possibility that autophagy could play quite different roles in different host cells and even within individual host cells during bacterial infection. Although further experiments are required, we hypothesize that, at the intracellular level, bacteria can employ "bet hedging" as a survival strategy. In conclusion, autophagy, NO production, and their interplay with RD1-encoded bacterial factors extend beyond their known roles in myelocytic cells to hLECs. Autophagy has been postulated as a pathway to target in the clinic in order to develop better vaccines and complement chemotherapy during tuberculosis treatment. Our data suggest that further studies are needed to validate this pathway as a good target, since, depending on the tissue and cell type infected, the autophagic response could be beneficial for $M$. tuberculosis.

During infection of lymph nodes with $M$. tuberculosis, lymphatic vessels not only play a structural role, regulating cell trafficking, but can also be infected and have an important cellautonomous immune function. We postulate that hLECs could be an important reservoir of $M$. tuberculosis during extrapulmonary and latent tuberculosis infection in humans. LECs in lymph nodes could represent an immune-privileged site, allowing persistence but also contributing to lymphangiogenic, inflammatory reactions during treatment. Our findings could explain well-documented clinical data in which patients with post-primary lymphatic tuberculosis are treated and cured but later reactivate.

\section{Methods}

Histology, immunohistochemistry, and analysis. Formalin-fixed paraffin-embedded tissue sections from patients diagnosed as tuberculosis culture positive and/or $\mathrm{AFB}^{+}$were selected for the study. Tissue sections were deparaffinized twice in xylene for 10 minutes and subsequently treated in $100 \%, 95 \%$, and $80 \%$ ethanol for 2 minutes at each gradient. Tissue sections were then placed into an antigen retrieval buffer (Access Super Antigen Solution, Menarini Diagnostics) in a decloaking chamber (Biocare Medical), incubated at 110 degrees for 10 minutes, and allowed to cool for 60 minutes. Sections were permeabilized in PBS-0.2\% Triton X-100 and incubated in blocking buffer (1\% BSA, $5 \%$ fetal calf serum in PBS) overnight at room temperature. Primary and secondary antibodies were tested for cross-reaction in samples from uninfected individuals. Primary (human antigens) and secondary antibodies were tested for cross-reaction with $M$. tuberculosis in samples that were $\mathrm{AFB}^{+}$. There was no cross-reaction of the antibodies used in this work with $M$. tuberculosis. In labeling with the anti-M. tuberculosis antibody, we obtained a distinct pattern of $M$. tuberculosis staining: typical shape and morphology, strong and specific association with granulomas, and macrophages within those granulomas. To validate the anti-M. tuberculosis antibody, we also stained consecutive sections with Ziehl Nielsen (ZN) and the antiM. tuberculosis antibodies. We observed a similar pattern of labeling distribution, and, in some groups of infected cells, we were able to correlate $\mathrm{ZN}$ with $M$. tuberculosis fluorescence staining (data not shown). Tissue sections were incubated at room temperature for 1 hour with primary antibodies diluted in PBS. After 3 washes in PBS, fluorescently labeled secondary antibodies were incubated for 1 hour at room temperature. Slides were washed 4 times with PBS and incubated for 10 minutes with DAPI, and then coverslips were applied using DAKO Mounting Medium. Samples were imaged with either an Olympus Fluoview 500 or a Leica SP5 laser scanning confocal microscope. Three-color images (red, green, and far red [pseudocolored as blue]) were acquired sequentially, followed by a DAPI image (gray) showing nuclei. Images were acquired and saved as TIFF format images. Images were processed using FIJI and Adobe Photoshop to subtract background and convolve images using a Gaussian function to reduce image noise. For semiquantitative analysis, at least 100 cells were 
counted per field of view, with an average of 200 cells. At least 10 fields of view were analyzed per section per sample.

Cell culture. Primary hLECs taken from inguinal lymph nodes (ScienCell Research Laboratories, 2500) were cultured according to the manufacturer's instructions up to passage 5. Briefly, T-75 flasks were coated with a solution of $2 \mu \mathrm{g} / \mathrm{cm}^{2}$ human fibronectin (Sigma-Aldrich, F1141) in sterile PBS (Life Technologies, 70013) and incubated overnight at $37^{\circ} \mathrm{C}$. hLECs were seeded at a density of 5,000 cells $/ \mathrm{cm}^{2}$ and cultured with endothelial cell medium (ECM) (ScienCell Research Laboratories, 1001) supplemented with $1 \%$ (v/v) endothelial cell growth supplement (ScienCell Research Laboratories, 1052) and 5\% (v/v) FBS (ScienCell Research Laboratories, 0025) at $37^{\circ} \mathrm{C}$ with $5 \%$ $\mathrm{CO}_{2}$. Culture medium was replaced every 2 days until the cells were $70 \%$ confluent; thereafter, the medium was replaced daily until $90 \%$ confluency, at which point the cells were used for experimentation or passaged according to the manufacturer's instructions. Different numbers of hLECs were seeded depending on the type of experiment, but generally a confluence of around 90\% was used for experimentation, and cells were seeded 16 hours prior to use. For confocal microscopy of fixed samples, hLECs were seeded at 20,000 cells per well in 300 $\mu \mathrm{l} \mathrm{ECM}$ onto fibronectin-treated $10-\mathrm{mm}$ diameter \#1.5 glass coverslips (Glaswarenfabrik Karl Hecht, 1001/10_15) placed into the wells of a 24-well plate. For CFU analysis or supernatant collection, hLECs were seeded at 20,000 cells per well in $300 \mu \mathrm{l} \mathrm{ECM}$ onto the fibronectintreated wells of a 24-well plate. For generating samples for Western blots or for samples for flat-cell embedding, hLECs were seeded at 300,000 cells per fibronectin-treated T- 25 flask in $5 \mathrm{ml} \mathrm{ECM}$. For live-cell imaging, 30,000 cells in $500 \mu \mathrm{l} \mathrm{ECM} \mathrm{per} \mathrm{fibronectin-treated}$ glass-bottom 35-mm live-cell dish (WillCo Wells, GWst-3512) were used, except in the case of live-cell imaging with CLEM, in which a much lower confluence was required (30\%-50\% was optimal). In this case, 10,000 cells in $500 \mu \mathrm{l} \mathrm{ECM}$ were seeded into fibronectin-treated 35-mm glass-bottom dishes (MatTek, P35G-2-14-CGRD). Information about isolating and culturing human macrophages is provided in the Supplemental Methods.

Bacterial strains and culture. M. tuberculosis $\mathrm{H} 37 \mathrm{Rv}$, M. tuberculosis $\mathrm{H} 37 \mathrm{Rv} \Delta \mathrm{RD} 1$, and M.tuberculosis $\mathrm{H} 37 \mathrm{Rv} \Delta \mathrm{RD} 1: \mathrm{RD} 1$ were transformed with the integrating EGFP expression vector pML1335 (provided by M. Niederweis, University of Alabama, Birmingham, Alabama, USA) to create the strains referred to in this study as M. tuberculosis WT, M. tuberculosis $\triangle \mathrm{RD} 1$, and $M$. tuberculosis $\triangle \mathrm{RD} 1$ :comp, respectively. M. bovis BCG strain Pasteur 1173P2 expressing GFP was provided by Brigitte Gicquel (Pasteur Institute, Paris, France). All strains were fully genome sequenced to confirm their identity prior to use. Mycobacteria were cultured in Middlebrook's 7H9 broth medium (Sigma-Aldrich, M0178) supplemented with 10\% (v/v) Middlebrook OADC (BD Biosciences, 212351) and $0.05 \%$ (v/v) Tween 80 (Sigma-Aldrich, P1754) and incubated at $37^{\circ} \mathrm{C}$ with rotation in $50-\mathrm{ml}$ Falcon tubes (cultures up to $10 \mathrm{ml}$ ) or roller bottles (cultures up to $50 \mathrm{ml}$ ). Mycobacteria were also cultured on Middlebrook's 7H11 agar medium (Sigma-Aldrich, M0428) supplemented with 10\% (v/v) Middlebrook OADC and incubated at $37^{\circ} \mathrm{C}$ for 2 to 3 weeks until colonies appeared.

Infection of hLECs with $M$. tuberculosis. hLECs were cultured and seeded onto fibronectin-treated plastic ware or glass coverslips as described above 16 hours prior to infection. Bacterial cultures were grown to midexponential phase $\left(\mathrm{OD}_{600}=0.6 \pm 0.2\right)$ as described above. $10 \mathrm{ml}$ of bacterial cultures were pelleted in a $15-\mathrm{ml}$ Falcon tube for 5 minutes at 2,000 $g$ in a benchtop centrifuge and then washed once with $10 \mathrm{ml}$ PBS and once with $10 \mathrm{ml}$ ECM, pelleting after each wash. An equivalent number of sterile 2.5- to 3.5-mm glass beads that matched the pellet size were added to the Falcon tube (usually $3-4$ beads) and then vigorously shaken for 1 minute to break up bacterial clumps. The bacteria were rinsed off of the side of the Falcon tube using $10 \mathrm{ml} \mathrm{ECM}$ and then spun at $300 \mathrm{~g}$ for 5 minutes. $8 \mathrm{ml}$ of the supernatant was then taken to a new 15-ml Falcon tube, and the measured $\mathrm{OD}_{600}$ was then diluted to 0.1 in ECM. Bacteria were added to hLECs at an MOI of 10 (calculated assuming that OD 0.1 contains $1 \times 10^{7}$ cells; data not shown) in a total volume of $300 \mu \mathrm{l} \mathrm{ECM} \mathrm{per} \mathrm{well} \mathrm{in} \mathrm{a} 24$-well plate or $1 \mathrm{ml} \mathrm{ECM}$ in a T-25 flask. The infection was incubated for 5 hours at $37^{\circ} \mathrm{C} 5 \% \mathrm{CO}_{2}$ before being carefully washed twice with $1 \mathrm{ml}$ PBS per well in a 24-well plate or $5 \mathrm{ml}$ PBS per T-25 flask to remove extracellular bacteria. ECM containing $10 \mu \mathrm{g} / \mathrm{ml}$ gentamicin sulfate (Fisher Scientific, BP918-1) (to prevent extracellular growth of $M$. tuberculosis in ECM; shown in Supplemental Figure 3E) and (where appropriate) $200 \mathrm{ng} / \mathrm{ml}$ recombinant human IFN- $\gamma$ (BD Biosciences, 554617) was added (1 ml per well in a 24-well plate or $5 \mathrm{ml} \mathrm{ECM} \mathrm{in} \mathrm{a} \mathrm{T-25} \mathrm{flask)} \mathrm{and} \mathrm{incubated} \mathrm{for} \mathrm{2,} \mathrm{24,} \mathrm{48,}$ and 72 hours. Where appropriate, the culture medium also contained the following chemical compounds: $10 \mathrm{mM}$ 3-MA (Sigma-Aldrich, M9281), $500 \mathrm{nM}$ rapamycin (LKT Laboratories Inc., R0161), and 250 $\mu$ M L-NMMA (Cayman Chemical, 80200).

CFU assay. After the indicated time point $(2,24,48$, or 72 hours after infection), $100 \mu \mathrm{l}$ of the supernatant from triplicate wells was taken and serially diluted in PBS in 10-fold steps until $10^{-2}$ dilution. 25 $\mu \mathrm{l}$ from each dilution (neat, $10^{-1}$, and $10^{-2}$ ) was plated in triplicate onto 7H11 agar plates. The rest of the supernatant was discarded, washed once with $1 \mathrm{ml} \mathrm{PBS}$, and then lysed with $500 \mu \mathrm{l}$ sterile water containing $0.05 \%$ Tween 80 to release the intracellular bacteria. After 30 minutes of incubation at room temperature, the lysed cell solution was diluted and samples were plated in exactly the same way as for the supernatant. Agar plates were incubated for 2 to 3 weeks at $37^{\circ} \mathrm{C}$ until colonies grew and were counted. Triplicate plates were averaged, and CFU were calculated and plotted as the average CFU per $\mathrm{ml}$ from triplicate wells.

Lactate dehydrogenase release assay. Viability of hLECs during infection with $M$. tuberculosis was measured using the lactate dehydrogenase release assay. Details are provided in the Supplemental Methods.

Field emission SEM. hLECs were seeded on fibronectin-pretreated 24-well plates on coverslip $\left(2 \times 10^{4}\right.$ cells per well $)$ and infected for 3 hours with $M$. bovis BCG (OD 0.01) or M. tuberculosis WT. At this time most of the bacteria were not completely internalized, as demonstrated by immunofluorescence with anti-mycobacterium antibody (Figure 2C). Samples were fixed with $2 \%$ glutaraldehyde and $5 \%$ formaldehyde in cacodylate buffer $\left(0.1 \mathrm{M}\right.$ cacodylate, $0.01 \mathrm{M} \mathrm{CaCl}_{2}$, $0.01 \mathrm{M} \mathrm{MgCl}_{2}, 0.09 \mathrm{M}$ sucrose, $\mathrm{pH}$ 6.9) for 1 hour on ice and washed with TE buffer (20 mM Tris, $1 \mathrm{mM}$ EDTA, pH 6.9), before dehydrating in a graded series of acetone $(10 \%, 30 \%, 50 \%, 70 \%, 90 \%, 100 \%)$ on ice for 15 minutes for each step. Samples were then critical-point dried with liquid $\mathrm{CO}_{2}$ (CPD 30, Bal-Tec) and covered with a gold film by sputter coating (SCD 500, Bal-Tec) before examination in a field emission SEM (Zeiss Merlin) using the Everhart-Thornley SE detector and the inlens SE detector in a 25:75 ratio at an acceleration voltage of $5 \mathrm{kV}$ at calibrated magnifications. Images were stored onto MO disks, and contrast and brightness were adjusted with Adobe Photoshop CS3 (Adobe Systems). 
$M R$ competition assay. hLECs were cultured in fibronectin-pretreated 24-well plates $\left(2 \times 10^{4}\right.$ cells per well) for 24 hours as described above. MR inhibition was performed by preincubating hLECs with $4 \mathrm{mg} / \mathrm{ml}$ of mannan (Sigma-Aldrich, M7504) for 2 hours (50). After that, the medium containing mannan was removed and new medium containing a suspension of M. bovis BCG (O.D. 0.01) was added. The samples were incubated for 5 hours at $37^{\circ} \mathrm{C}$. To verify whether mannan itself could interact with $\mathrm{BCG}$ and influence the internalization, BCG (O.D. 0.01) were pretreated with mannan solution $(4 \mathrm{mg} / \mathrm{ml})$ for 2 hours before being added to the untreated hLECs and incubated for more 5 hours at $37^{\circ} \mathrm{C}$. Mycobacterial uptake was analyzed by CFU as described above. To confirm that BCG was internalized, an anti-mycobacteria antibody was used without prior permeabilization of the sample.

Indirect immunofluorescence. hLECs infected on coverslips were infected as described above, and, at the indicated time points, cells were fixed with $3 \%$ methanol-free PFA (Electron Microscopy Sciences, 15710) in PBS for 24 hours at $4^{\circ} \mathrm{C}$. Coverslips were then quenched with $50 \mathrm{mM} \mathrm{NH}_{4} \mathrm{Cl}$ (Sigma-Aldrich, A9434) in PBS for 15 minutes at room temperature and permeabilized with 0.01\% saponin (Sigma-Aldrich, 84510) 1\% BSA (Sigma-Aldrich, A3912) in PBS for 10 minutes. The cells were then washed once with PBS for 5 minutes, and the primary antibody was diluted in PBS containing $0.01 \%$ saponin and $1 \%$ BSA. The coverslips were placed onto a sheet of parafilm on the bench, and 30 to $50 \mu$ of antibody dilution was added on top of the coverslip and incubated in the dark for the appropriate time (see below). Subsequently, the coverslips were washed 3 times in PBS for 5 minutes each time, and then the secondary antibody was added in the same way as the primary antibody for 1 hour at room temperature. After 3 more washes with PBS, an optional step to stain for F-actin using a 1:250 dilution of either rhodamine-phalloidin (Biotium, 00027) or Alexa Fluor 633-phalloidin (Life Technologies, A22284) was performed for 20 minutes at room temperature. After another 3 PBS washes, nuclear staining was performed using 300 nM DAPI (Life Technologies, D3571) in PBS for 10 minutes. One final wash with PBS was performed before mounting the coverslips on glass slides using DAKO mounting medium (DAKOCytomation, S3023).

Confocal microscopy and image acquisition. Confocal imaging (of fixed and live samples) was performed using a Leica SP5 AOBS Laser Scanning Confocal Microscope (Leica Microsystems) equipped with an environmental control chamber (EMBL, provided under the EMBL Enterprise Management Technology Transfer $\mathrm{GmbH}$ ), providing a constant temperature of $37^{\circ} \mathrm{C} 5 \% \mathrm{CO}_{2}$, a $\mathrm{HC}$ PL APO CS2 $63.0 \times / 1.4$ OIL UV objective, a UV laser (405 nm), an argon laser (488 nm), a DPSS laser (561 nm), a HeNe laser $(633 \mathrm{~nm})$, and one PMT and two HyD detectors. Immersion oil used for fixed samples was Leica Type F (Leica Microsystems, 11513859) and for live-cell imaging was Cargille Type 37 (Cargille Labs). Image acquisition for fixed samples was performed using the following settings: scanning mode xyz, sequential acquisition, pixel resolution of $1,024 \times 1,024$, scanner frequency of 200 $\mathrm{Hz}$, line averaging of 4 , and zoom of 1 . The settings for live-cell imaging were the same except for scanning mode xyzt (temporal control varied, but frames were usually acquired at 5- to 10-minute intervals), line averaging of 2 , and zoom of 2 .

Image analysis. All image analysis was performed using FIJI (NIH). Leica SP5 files (.lif) were opened using the BioFormats plug-in of FIJI. Custom-written macros were used to analyze the images as follows: (a) To measure intracellular bacterial growth (measuring the bacterial
GFP signal per hLEC), a region of interest (ROI) (i.e., a hLEC containing intracellular GFP bacteria [green channel]) was drawn using the line tool within FIJI, using the actin cytoskeleton (red or far-red channel) as a guide. The ROI was duplicated, and anything outside the ROI was cleared. Then, the images were split into their constitutive color channels, and the green channel (corresponding to the GFP signal from the bacteria) was subjected to a pixel threshold. The bacteria were selected, and the RAWIntDen measurement was taken (this is the sum of all the pixels originating from the GFP signal, which correlates with the number of intracellular bacteria). (b) To measure the association of a marker (e.g., cathepsin D) with individual bacterial particles (i.e., individual bacterial phagosomes/entities), The multichannel image (blue, DAPI; green, GFP; red, Alexa Fluor 546; and far red, Alexa Fluor 633-phalloidin) was split into individual channels, and the green channel was subjected to a pixel threshold. A mask corresponding to all the bacteria was created, and then the Analyze Particles function of FIJI (size $=0.5$-infinity, circularity $=0-1$ ) was used to outline all the bacterial "particles" in the field of view. The mean pixel intensity in the red channel (corresponding to the secondary antibody) within the particle was measured. All data were plotted and analyzed using Microsoft Excel 2010 (Microsoft) and GraphPad Prism 6 (GraphPad Software Inc.).

siRNA KD of ATG5. To KD ATG5 protein levels, hLECs were seeded at 10,000 cells per well in fibronectin-coated wells of a 24-well plate. After attachment, $0.5 \mu \mathrm{l}$ of $20 \mu \mathrm{M}$ siRNA (SMARTpool ON-TARGETplus, GE Dharmacon; ATG5, L-004374-00; Nontargeting, D-001810-10) was diluted in $94.5 \mu \mathrm{l}$ serum-free medium, which was then mixed with $5 \mu \mathrm{l}$ of HiPerFect Transfection Reagent (Qiagen, 301705) for 15 minutes at room temperature, and then added to the cells in a dropwise manner. The cells were incubated for 4 hours, and then $200 \mu$ l of complete ECM was added to the wells. After 24 hours, this process was repeated to achieve a second round of $\mathrm{KD}$, and, after a further 48 hours, the cells were ready to infect as described above. After infection, a third round of KD was performed, and samples were collected 48 hours after infection.

Western blotting. To prepare the samples, hLECs were infected in a T-25 flask for each condition required. At the desired time point, cells were washed once with $5 \mathrm{ml}$ PBS, and then the cells were scraped into $1 \mathrm{ml}$ cold PBS and transferred to a microcentrifuge tube. The cells were pelleted by centrifugation at $4^{\circ} \mathrm{C}$ at $17,000 \mathrm{~g}$ for 5 minutes, and the supernatant was removed. The pellets were resuspended in $100 \mu \mathrm{l}$ cold Nonidet-P40 (NP40) lysis buffer (consisting of $150 \mathrm{mM} \mathrm{NaCl}, 1 \%$ NP40, 50 mM Tris-HCl, protease inhibitors (cOmplete Mini Tablets, Roche) and incubated on ice for 40 minutes. To this, $33 \mu \mathrm{l}$ of NuPage LDS sample buffer (Life Technologies, NP008) was added, and the samples were heated to $95^{\circ} \mathrm{C}$ for 20 minutes. Finally, $15 \mu \mathrm{l}$ of NuPage reducing agent (Life Technologies, NP0009) was added to each sample before samples were frozen at $-20^{\circ} \mathrm{C}$. Samples were thawed for SDS-PAGE, and, prior to gel loading, the samples and ladder were heated to $80^{\circ} \mathrm{C}$ for 10 minutes. Typically, $15 \mu$ of the cell lysate was loaded into each well of a 10 -well precast NuPAGE $4 \%$ to $12 \%$ gradient Bis-Tris gel (Life Technologies, NP0321BOX) along with $6 \mu \mathrm{l}$ of BLUeye Prestained Protein Ladder (Geneflow Ltd., S6-0024). Gels were run in 1x NuPAGE MES SDS Running Buffer (Life Technologies, NP0002) with 1x NuPAGE antioxidant (Life Technologies, NP0005) added. The gels were run for 35 minutes at a constant $200 \mathrm{~V}$. The cassettes were cracked open, and the membrane was equilibrated in $20 \%$ 
ethanol prior to transfer onto a nitrocellulose membrane using the iBlot 2 Dry Blotting System (Life Technologies) according to the manufacturer's instructions (using a 7-minute transfer program). To perform the Western Blot, the nitrocellulose membrane was then blocked in a solution of $5 \%(\mathrm{w} / \mathrm{v})$ milk (Sigma-Aldrich, 70166) and $0.05 \%(\mathrm{v} / \mathrm{v})$ Tween20 (MP Biomedicals, TWEEN201) in PBS overnight at $4^{\circ} \mathrm{C}$ with rocking. The membranes were cut according to the size of the protein of interest and then incubated with primary antibody overnight at $4{ }^{\circ} \mathrm{C}$ with rocking. The membrane was washed 3 times for 5 minutes each in PBS-T (0.05\% [v/v] Tween20 in PBS) and incubated with secondary HRP-conjugated antibodies. $\beta$-Actin was used as loading control. Bands were visualized using the Amersham ECL Western Blotting Detection Reagent (GE Healthcare, RPN2106) and exposed on Amersham Hyperfilm ECL (GE Healthcare, 28906837) using a developer set for a 2-minute exposure time. Bands were quantified using FIJI software and myImageAnalysis software (Thermo Fisher Scientific).

Ultrastructural analysis. The protocol for resin embedding was adapted from ref. 51 . Samples were fixed by adding warm $2 \%$ glutaraldehyde (EMS) in $200 \mathrm{mM}$ HEPES, $\mathrm{pH}$ 7.4, directly to the cell culture medium at a 1:1 volume ratio. After 5 minutes, the mixture of the fixative and medium was replaced with $1 \%$ glutaraldehyde in HEPES buffer, and the samples were incubated overnight at $4^{\circ} \mathrm{C}$. Cell monolayers were post-fixed with $2 \%$ osmium tetroxide (EMS) solution containing $1.5 \%$ potassium ferricyanide for 1 hour on ice and then stained with $0.5 \%$ tannin for 20 minutes and with $1.5 \%$ aqueous uranyl acetate (EMS) for 1 hour. Next, cells were dehydrated at room temperature using a graded ethanol series $(70 \%, 80 \%, 90 \%, 95 \%, 100 \%)$ and then detached from plastic with propylene oxide and washed several times, followed by gradual infiltration with Spurr's resin (Polysciences) over 2 days. Ultrathin sections $(70 \mathrm{~nm})$ were cut with a Leica ultramicrotome Ultracut EM UCT (Leica Microsystems) and contrasted with $0.2 \%$ lead citrate. Cells were examined with a CM100 transmission electron microscope (FEI). The images were recorded digitally with a Quemesa TEM CCD camera (Olympus Soft Imaging Solutions) and iTEM software (Olympus Soft Imaging Solutions). For subcellular localization analysis, fractions of phagosomal, cytosolic, autophagic, and lysosomal bacteria were determined. At least 15 different infected cells per sample were imaged at $\times 3,900$ magnification by systematic and random sampling. Cross points of the stereological test grid over bacteria were counted with regard to the subcellular localization of bacteria, which was determined from images taken at minimum magnification of $\times 8,900$. Fractions were calculated from total counts per sample. The following criteria were followed for the assessment of subcellular localization: (a) phagosomal bacteria were, at least partially, tightly lined by a phospholipid bilayer, representing the phagosomal membrane; (b) cytosolic bacteria were surrounded by ribosomes, representing the cytoplasm with no indication of the phagosomal membrane; (c) autophagic bacteria were enveloped by double or multiple membrane structures; and (d) lysosomal bacteria resided in loose vesicles containing additional structures. Details about immunogold labeling on Tokuyasu thawed cryosections can be found in the Supplemental Methods.

CLEM using a 3View system. hLECs were seeded onto fibronectintreated MatTek dishes at 30\% to 50\% confluence (approximately 10,000 cells) as described above. While the cells were in suspension, $10 \mu$ Premo Autophagy Sensor LC3B-RFP BacMam 2.0 (Life Technologies, P36236) was added to transduce the cells prior to overnight incubation at $37^{\circ} \mathrm{C}$ with $5 \% \mathrm{CO}_{2}$. hLECs were infected with $M$. tuberculosis WT as described above; after 5 hours of infection, the dishes were washed 3 times with PBS, and $1 \mathrm{ml} \mathrm{ECM}$ was added, containing $10 \mu \mathrm{l} \mathrm{BacMam}$ 2.0 LC3B-RFP to boost transduction. The dishes were securely fastened into a custom-made dish holder for the stage of a Leica SP5 confocal microscope. Imaging conditions were as described above, although the zoom was set to 2 and the acquisition software (Leica LAS AF) was set to 2-hour frames for the first 12 hours, with 30-minute frames thereafter. Z-stacks of 5 slices were taken at each time point, with a $1.19-\mu \mathrm{m}$ thickness. For correlative light and serial block-face SEM, time-lapse and confocal fluorescence imaging were performed on cells of interest, and the sample was then fixed in $4 \%$ $\mathrm{PFA} / 2.5 \%$ glutaraldehyde in $0.1 \mathrm{M}$ phosphate buffer ( $\mathrm{pH}$ 7.4) for 24 hours at $4^{\circ} \mathrm{C}$. Using a modified NCMIR protocol (52), coverslips were post-fixed in $2 \%$ osmium tetroxide $1.5 \%$ potassium ferrocyanide for 1 hour on ice, incubated in 1\% w/v thiocarbohydrazide for 20 minutes before a second staining with $2 \%$ osmium tetroxide, and then incubated overnight in $1 \%$ aqueous uranyl acetate at $4^{\circ} \mathrm{C}$. Cells were stained with Walton's lead aspartate for 30 minutes at $60^{\circ} \mathrm{C}$ and dehydrated through an ethanol series on ice, incubated in a 1:1 propylene oxide/ Durcupan resin mixture, and embedded in Durcupan resin according to the manufacturer's instructions (TAAB Laboratories Equipment Ltd.). Images were collected using a 3View 2XP system (Gatan Inc.) mounted on a Sigma VP Scanning Electron Microscope (Carl Zeiss). Images were collected at $1.8 \mathrm{kV}$ using the high-current setting, with a $20-\mu \mathrm{m}$ aperture, at $10 \mathrm{~Pa}$ chamber pressure, and a $2-\mu \mathrm{s}$ dwell time. The data set was $24.25 \times 24.25 \times 3.35 \mu \mathrm{m}$ in xyz, consisting of 68 serial images of 50-nm thickness and a pixel size of $2.96 \times 2.96 \mathrm{~nm}$. The total volume of the data set was approximately $1,970 \mu \mathrm{m}^{3}$. The data set was segmented manually using Amira 6.0 (FEI Visualization Sciences Group). M. tuberculosis (green) and the exterior of the M. tuberculosiscontaining compartment (red) were manually selected by morphology with reference to the correlative fluorescence data set. Where the bacteria closely abutted the surface, the segmentation was extended by approximately $270 \mathrm{~nm}$ beyond the true surface position to prevent artefactual holes from appearing in the reconstructed surface. A movie of the serial images and 3D-rendered model was created in Amira and compressed in QuickTime 7 (Apple Inc.) using H264 compression.

Antibodies. Antibodies used for immunofluorescence, immunohistochemistry, and Western blot are provided in the Supplemental Methods.

Statistics. Results are expressed as mean \pm SEM. All statistical analyses were performed in Prism 6 (GraphPad Software Inc.). Means between 2 groups were compared using 2-tailed Student's $t$ tests, and means among 3 or more groups were compared using 1-way ANOVA with Tukey's multiple comparisons tests. A $P$ value less than 0.05 was considered significant.

Study approval. The study was performed using excised cervical lymph node tissue stored within the Department of Anatomical Pathology at Groote Schuur Hospital. All of these biopsies were taken for clinical indications. Residual paraffin-embedded blocks of these specimens were stored for further processing. This study complied with the Declaration of Helsinki (2008), and ethics approval was obtained from the University of Cape Town Human Research Ethics Committee (REC187/2013). Informed consent was waived, as this was a retrospective study of formalin-fixed paraffin-embedded tissue samples collected during the course of routine clinical practice. Patient identifiers were unavailable to investigators. 


\section{Author contributions}

TRL, CDSCW, UR, MRGR, SB, MR, HW, and MGG conducted experiments. TRL, CDSCW, UR, MRGR, SB, CRD, LMC, GG, HW, and RJW analyzed data. MGG, TRL, CDSCW, UR, and MRGR made the figures. MGG, CDSCW, and TRL wrote the first draft of the manuscript, and all authors contributed to the final version.

\section{Acknowledgments}

We thank Cecilie Nesset for her technical assistance with electron microscopic analysis, John M. Lucocq for advice on stereological analysis, Steve Coade for technical support, Radma Mahmood and Radhika Anand for excellent assistance with histology, Bernard Hoflack for the anti-cathepsin antibody, Michael Niederweis for integrative EGFP plasmids, Douglas Young and Suzanne Hingley-Wilson for mycobacterial strains, Laura Schnettger for help with the FIJI macro writing, and the EM lab at the Institute of Biosciences, University of Oslo, for access to instruments. We thank Serge Mostowy for helpful discussions and critical reading of this manuscript. This work was supported by the Coordination for the Improvement of Higher Education (0561-11-8 to C. de Souza Carvalho-Wodarz); a Helmholtz Young Investigator grant (VH-NG-527 to M.G. Gutierrez); the Francis Crick Institute, which receives its core funding principally from Cancer Research UK, the UK Medical Research Council (MC_UP_1202/11 to M.G. Gutierrez and U1175.02.002.00014 to R.J. Wilkinson), and the Wellcome Trust (104803 and 084323 to R.J. Wilkinson); The Claude Leon Foundation; the European Commission (FP7-PEOPLE-2011IRSES to R.J. Wilkinson); and the German Research Council (DFG SPP1580 to M.G. Gutierrez and G. Griffiths).

Address correspondence to: Maximiliano G. Gutierrez, The Francis Crick Institute, Mill Hill Laboratory, The Ridgeway, Mill Hill, London, NW7 1AA, United Kingdom. Phone: 44.0.208.816.2225; E-mail: maximiliano.gutierrez@crick.ac.uk.
1. Sharma SK, Mohan A. Extrapulmonary tuberculosis. Indian J Med Res. 2004;120(4):316-353.

2. Behr MA, Waters WR. Is tuberculosis a lymphatic disease with a pulmonary portal? Lancet Infect Dis. 2014;14(4):250-255.

3. O'Garra A, Redford PS, McNab FW, Bloom CI, Wilkinson RJ, Berry MP. The immune response in tuberculosis. Annu Rev Immunol. 2013;31:475-527.

4. Cooper AM, Dalton DK, Stewart TA, Griffin JP, Russell DG, Orme IM. Disseminated tuberculosis in interferon gamma gene-disrupted mice. JExp Med. 1993;178(6):2243-2247.

5. Al-Muhsen S, Casanova JL. The genetic heterogeneity of mendelian susceptibility to mycobacterial diseases. J Allergy Clin Immunol. 2008;122(6):1043-1051.

6. Flynn JL, Chan J, Triebold KJ, Dalton DK, Stewart TA, Bloom BR. An essential role for interferon gamma in resistance to Mycobacterium tuberculosis infection. J Exp Med.1993;178(6):2249-2254.

7. Desvignes L, Ernst JD. Interferon- $\gamma$-responsive nonhematopoietic cells regulate the immune response to Mycobacterium tuberculosis. Immunity. 2009;31(6):974-985.

8. Forrellad MA, et al. Virulence factors of the Mycobacterium tuberculosis complex. Virulence. 2013;4(1):3-66.

9. Pym AS, et al. Recombinant BCG exporting ESAT- 6 confers enhanced protection against tuberculosis. Nat Med. 2003;9(5):533-539.

10. Hsu T, et al. The primary mechanism of attenuation of bacillus Calmette-Guerin is a loss of secreted lytic function required for invasion of lung interstitial tissue. Proc Natl Acad Sci U S A. 2003;100(21):12420-12425.

11. Stanley SA, Raghavan S, Hwang WW, Cox JS. Acute infection and macrophage subversion by Mycobacterium tuberculosis require a specialized secretion system. Proc Natl Acad Sci U S A. 2003;100(22):13001-13006.

12. Lewis KN, et al. Deletion of RD1 from Mycobacterium tuberculosis mimics bacille Calmette-Guerin attenuation. J Infect Dis. 2003;187(1):117-123.

13. van der Wel N, et al. M. tuberculosis and M. leprae translocate from the phagolysosome to the cytosol in myeloid cells. Cell. 2007;129(7):1287-1298.
14. Watson RO1, Manzanillo PS, Cox JS. Extracellular M. tuberculosis DNA targets bacteria for autophagy by activating the host DNA-sensing pathway. Cell. 2012;150(4):803-815.

15. Simeone R, et al. Phagosomal rupture by Mycobacterium tuberculosis results in toxicity and host cell death. PLoS Pathog. 2012;8(2):e1002507.

16. Hernández-Pando R, et al. Persistence of DNA from Mycobacterium tuberculosis in superficially normal lung tissue during latent infection. Lancet. 2000;356(9248):2133-2138.

17. Pepper MS, Skobe M. Lymphatic endothelium: morphological, molecular and functional properties. JCell Biol. 2003;163(2):209-213.

18. Podgrabinska S, Braun P, Velasco P, Kloos B, Pepper MS, Skobe M. Molecular characterization of lymphatic endothelial cells. Proc Natl Acad Sci US A. 2002;99(25):16069-16074.

19. Mai J, Virtue A, Shen J, Wang H, Yang XF. An evolving new paradigm: endothelial cells - conditional innate immune cells. J Hematol Oncol. 2013;6:61.

20. Jurisic G, Detmar M. Lymphatic endothelium in health and disease. Cell Tissue Res. 2009;335(1):97-108.

21. Tewalt EF, Cohen JN, Rouhani SJ, Engelhard VH. Lymphatic endothelial cells - key players in regulation of tolerance and immunity. Front Immunol. 2012;3:305.

22. Card CM, Yu SS, Swartz MA. Emerging roles of lymphatic endothelium in regulating adaptive immunity. J Clin Invest. 2014;124(3):943-952.

23. Park SM, et al. Mapping the distinctive populations of lymphatic endothelial cells in different zones of human lymph nodes. PLoS One. 2014;9(4):e94781.

24. Schlesinger LS. Macrophage phagocytosis of virulent but not attenuated strains of Mycobacterium tuberculosis is mediated by mannose receptors in addition to complement receptors. JImmunol. 1993;150(7):2920-2930.

25. Nörder M, Gutierrez MG, Zicari S, Cervi E, Caruso A, Guzmán CA. Lymph node-derived lymphatic endothelial cells express functional costimulatory molecules and impair dendritic cell-induced allogenic T-cell proliferation. FASEB J. 2012;26(7):2835-2846.

26. Tobin DM, et al. Host genotype-specific therapies can optimize the inflammatory response to mycobacterial infections. Cell. 2012;148(3):434-446.

27. Fenaroli F, et al. Nanoparticles as drug delivery system against tuberculosis in zebrafish embryos: direct visualization and treatment. ACS Nano. 2014;8(7):7014-7026.

28. Russell DG. Mycobacterium tuberculosis and the intimate discourse of a chronic infection. Immunol Rev. 2011;240(1):252-268.

29. Houben D, et al. ESX-1-mediated translocation to the cytosol controls virulence of mycobacteria. Cell Microbiol. 2012;14(8):1287-1298.

30. Gutierrez MG, Master SS, Singh SB, Taylor GA, Colombo MI, Deretic V. Autophagy is a defense mechanism inhibiting BCG and Mycobacterium tuberculosis survival in infected macrophages. Cell. 2004;119(6):753-766.

31. Collins CA, De Mazière A, van Dijk S, Carlsson F, Klumperman J, Brown EJ. Atg5-independent sequestration of ubiquitinated mycobacteria. PLoS Pathog. 2009;5(5):e1000430.

32. Pankiv S, et al. p62/SQSTM1 binds directly to Atg8/LC3 to facilitate degradation of ubiquitinated protein aggregates by autophagy. J Biol Chem. 2007;282(33):24131-24145.

33. Pegu A, Flynn JL, Reinhart TA. Afferent and efferent interfaces of lymph nodes are distinguished by expression of lymphatic endothelial markers and chemokines. Lymphat Res Biol. 2007;5(2):91-103.

34. Basaraba RJ, Smith EE, Shanley CA, Orme IM. Pulmonary lymphatics are primary sites of Mycobacterium tuberculosis infection in guinea pigs infected by aerosol. Infect Immun. 2006;74(9):5397-5401.

35. Datta $M$, et al. Anti-vascular endothelial growth factor treatment normalizes tuberculosis granuloma vasculature and improves small molecule delivery. Proc Natl Acad Sci U S A. 2015;112(6):1827-1832.

36. Harding J, Ritter A, Rayasam A, Fabry Z, Sandor M. Lymphangiogenesis is induced by mycobacterial granulomas via vascular endothelial growth 
factor receptor-3 and supports systemic T-cell responses against mycobacterial antigen. $\mathrm{Am} \mathrm{J}$ Pathol. 2015;185(2):432-445.

37. Kerjaschki $D$. The lymphatic vasculature revisited. JClin Invest. 2014;124(3):874-877.

38. Marttila-Ichihara F, et al. Macrophage mannose receptor on lymphatics controls cell trafficking. Blood. 2008;112(1):64-72.

39. Nathan CF, Murray HW, Wiebe ME, Rubin BY. Identification of interferon $\gamma$ as the lymphokine that activates human macrophage oxidative metabolism and antimicrobial activity. JExp Med. 1983;158(3):670-689.

40. Kumar D, et al. Genome-wide analysis of the host intracellular network that regulates survival of Mycobacterium tuberculosis. Cell. 2010;140(5):731-743.

41. Romagnoli A, et al. ESX-1 dependent impairment of autophagic flux by Mycobacterium tuberculosis in human dendritic cells. Autophagy. 2012;8(9):1357-1370.

42. Fine KL, Metcalfe MG, White E, Virji M, Karls RK, Quinn FD. Involvement of the autophagy pathway in trafficking of Mycobacterium tuberculosis bacilli through cultured human type II epithelial cells. Cell Microbiol. 2012;14(9):1402-1414.

43. Pacheco SA, Powers KM, Engelmann F, Messaoudi I, Purdy GE. Autophagic killing effects against by alveolar macrophages from young and aged rhesus macaques. PLoS One. 2013;8(6):e66985.

44. Birmingham CL, Canadien V, Kaniuk NA, Steinberg BE, Higgins DE, Brumell JH. Listeriolysin $\mathrm{O}$ allows Listeria monocytogenes replication in macrophage vacuoles. Nature. 2008;451(7176):350-354.

45. Vázquez CL, et al. Experimental selection of long-term intracellular mycobacteria. Cell Microbiol. 2014;16(9):1425-1440.

46. Shaul PW. Regulation of endothelial nitric oxide synthase: location, location, location. Annu Rev Physiol. 2002;64:749-774.

47. Koedel U, Paul R, Winkler F, Kastenbauer S, Huang PL, Pfister HW. Lack of endothelial nitric oxide synthase aggravates murine pneumococcal meningitis. J Neuropathol Exp Neurol. 2001;60(11):1041-1050.

48. Yang $\mathrm{Z}$, et al. Female resistance to pneumonia identifies lung macrophage nitric oxide synthase- 3 as a therapeutic target. Elife. 2014;3:e03711.

49. Mattila JT, et al. Microenvironments in tuberculous granulomas are delineated by distinct populations of macrophage subsets and expression of nitric oxide synthase and arginase isoforms. JImmunol. 2013;191(2):773-784.

50. Kang PB, et al. The human macrophage mannose receptor directs Mycobacterium tuberculosis lipoarabinomannan-mediated phagosome biogenesis. JExp Med. 2005;202(7):987-999.

51. Rohde KH, Veiga DF, Caldwell S, Balázsi G, Russell DG. Linking the transcriptional profiles and the physiological states of Mycobacterium tuberculosis during an extended intracellular infection. PLoS Pathog. 2012;8(6):e1002769.

52. Deerinck TJ, Bushong EA, Thor A, Ellisman MH. NCMIR methods for 3D EM: A new protocol for preparation of biological specimens for serial block face scanning electron microscopy. Microscopy.2010;6-8. http://gatan.actonservice. com/acton/attachment/11413/f-017e/1/-/-/-/-/ sbfsem\%20sample\%20prep\%20protocol. pdf?modal=1. Accessed on February 9, 2016. 